\title{
Linear elastic fracture simulation directly from CAD: 2D NURBS-based implementation and role of tip enrichment
}

\author{
X. Peng ${ }^{1}$, E. Atroshchenko ${ }^{2}$, P. Kerfriden ${ }^{1}$, S. P. A. Bordas ${ }^{3,1,4}$ \\ ${ }^{1}$ Institute of Mechanics Materials and Advanced Manufacturing, Cardiff University, CF24 \\ $3 \mathrm{AA}, \mathrm{UK}$ \\ ${ }^{2}$ Department of Mechanical Engineering, University of Chile, Santiago, 8370448, Chile \\ ${ }^{3}$ Université du Luxembourg, Faculté des Sciences, de la Technologie et de la Communication, \\ 6 rue Richard Coudenhove-Kalergi, L-1359 Luxembourg - Research Unit in Engineering \\ Science Campus Kirchberg, G 007 \\ ${ }^{4}$ University of Western Australia, 35 Stirling Hwy, Crawley, WA 6009, Australia
}

\begin{abstract}
We propose a method for simulating linear elastic crack growth through an isogeometric boundary element method directly from a CAD model and without any mesh generation. To capture the stress singularity around the crack tip, two methods are compared: (1) a graded knot insertion near crack tip; (2) partition of unity enrichment. A well-established CAD algorithm is adopted to generate smooth crack surfaces as the crack grows. The $M$ integral and $J_{k}$ integral methods are used for the extraction of stress intensity factors (SIFs). The obtained SIFs and crack paths are compared with other numerical methods.
\end{abstract}

Key Words: Isogeometric analysis; NURBS; Linear elastic fracture; Boundary element method; Crack growth.

\section{Introduction}

Meshing and remeshing is one of the most human interactive task in fracture simulation. Most if not all, commercial codes do not offer completely automatic approach for industrial fracture simulations. The difficulties associated with computational fracture mechanics have various sources. First, given a CAD model of the component, a suitable mesh has to be generated, usually orders of magnitude finer in the region(s) where cracks are introduced, than the mesh

\footnotetext{
${ }^{\top}$ Corresponding author. Tel: $+56(2) 9784415$ Fax: $+56(2) 6896057$

E-mail address:eatroshch@gmail.com
} 
used for stress analysis. Second, the discontinuities engendered by the cracks must be followed during crack propagation. Third, the discretization must be able to reproduce the large gradients (singularities in the case of linear elastic fracture mechanics (LEFM)). This requirement, combined with that of capturing discontinuities as they evolve implies that relatively fine meshes must be continuously regenerated as cracks propagate. Fourth, reliable and general fracture models remain elusive. For LEFM, the Paris law or its cousins are commonly used. Such laws compute the increment in crack advance as a proportional to some power $m(m>1)$ of the stress intensity factor (SIF). A small error $\varepsilon_{S I F}$ in the SIF thus leads to an accumulated error scaling as $m \varepsilon_{S I F}$ at each of the tens of thousands of crack growth steps required for each simulation.

Consequently, various approaches have been developed to overcome, or at least alleviate those difficulties as listed in Table 1.

\begin{tabular}{|c|c|}
\hline CAD \& Mesh & IGA [1], IGABEM [2][3], automatic remeshing [4] \\
\hline Discontinuities & Meshless [5], XFEM [6], BEM [7] \\
\hline Large gradients \& Singularities & XFEM [6], XIGA [8][9], cohesive IGA [10] \\
\hline Models \& Error & $\begin{array}{c}\text { XFEM error estimators [11][12], XSPR [13][14] } \\
\text { homogenization \& multiscale fracture modeling [15][16] }\end{array}$ \\
\hline
\end{tabular}

Table 1: Difficulties associated with crack modeling and main remedies, see also Rabczuk et al [17]

The boundary element method (BEM) has been applied for simulating fracture problems for several decades due to the fact that (1) the governing equations are accurately satisfied in the domain interior with the use of fundamental solution and the discretization of the geometry and approximation of the quantities of interest only occur over the boundary. BEM is in particular able to capture the stress concentration or singularity better than domain integration methods such as the finite element method (FEM) [18]; (2) the dimensionality of the problem in BEM is reduced by one and only the boundary geometry and discretization must be modified when cracks evolve, which simplifies the remeshing procedure. An important issue for modeling fracture using BEM is the degeneration of the system matrix when the source points are placed on overlapping crack surfaces. Much work was done to address this problem. Blandford et al [19] used the multi-region method to model crack problems by dividing the domain into sub-domains along the crack surface and introducing artificial boundaries. This approach is cumbersome in dealing with multiple cracks and crack propagation problems. Synder and Cruse [20] developed a modified fundamental kernel for infinite domains containing flat, traction free cracks in a $2 \mathrm{D}$ 
mixed-mode problem. However, the proposed kernel is limited to flat cracks. The most popular approach to overcome the degeneration of the system is to prescribe displacement boundary integral equation (BIE) on one crack surface and traction BIE on the other crack surface. The method is called dual boundary element method (DBEM) [21]. DBEM provides an efficient way to model cracks of arbitrary 1D and 2D geometries [22][23][7][24]. Another approach is known as the displacement discontinuity method (DDM) [25], which is mostly suitable for problems with symmetry. In this method, the two overlapping crack surfaces are replaced by one of the surfaces, which decreases the computational model size. Also the displacement and traction discontinuities on the crack surface are used as primary quantities instead of displacement and traction on the two crack surfaces in DBEM. in such a case, even a single traction BIE can be used for fracture problems [26]. DDM was later proved to be a special case of DBEM by Partheymüller et al [27], who also extended the application of DDM from symmetric loaded cracks to asymmetric loaded cracks. However the displacement field on the crack surface is indirect since only displacement discontinuity is obtained. Additional postprocessing needs to be done to retrieve the displacement solution which increases the implementation complexity and the computational burden.

Another branch of work has focused on the Galerkin formulation of BEM, particularly symmetric Galerkin BEM (SGBEM), for fracture mechanics, which is primarily based on DDM [28][29][30]. In Galerkin formulations, the error estimation theory is well developed and the boundary continuity requirement is relaxed to be $C^{0}$ for hyper-singular BIE due to the weak form [31]. However, double integrals must be evaluated which makes the method slower but also more stable than the collocation BEM. In order to make the crack modeling more efficient for large scale problems, hybrid BEM-FEM schemes were proposed [32][33][34]. The general idea is to subdivide the cracked domain into two sub-domains, the BEM sub-domain and the FEM sub-domain, to take advantage of both methods. Some other methods like boundary element-free method [35] and the scaled boundary finite element method (SBFEM) [36] were also proposed and applied to fracture modeling .

The accurate evaluation of stress intensity factors (SIFs) plays a pivotal role in crack growth modeling. Due to the $1 / \sqrt{r}$ stress singularity in the vicinity of the crack tip, special care should be taken in the numerical methods in order to absolutely obtain more accurate SIFs. One approach to capture the asymptotics of the displacement and stress fields in the vicinity of 
a crack is the use of special crack tip elements; for example, quarter-point elements [37][38], which can exactly represent the $1 / \sqrt{r}$ singularity in the near-tip stress field and allow a direct extraction of the SIFs [39]. Another possibility is the hybrid crack element, developed in both the FEM and the BEM communities [40][41], which introduces asymptotic behavior of the stress field around crack tip into the tip-element so that the SIFs can be computed directly and accurately.

The virtual crack closure technique (VCCT) based on the Irwin's integral of strain energy release rates, is a common method to extract SIFs in both the FEM and the BEM, and has recently been extended to extended finite element method (XFEM) and extended element-free Galerkin method (XEFG) [42][43]. Since the near-tip singular behavior is already known as Williams' solution, the idea is to remove the singularity and extract the SIFs directly[44]. However, the Williams' solution is only valid in the 'near-tip' region. The determination of this 'near-tip' region for simulation is ambiguous in practical problems.

$J$ integral based methods are regarded as very accurate approaches to extract SIFs in both FEM and BEM communities. Different approaches to the extraction of $J_{1}(J)$ were developed, such as the symmetric and asymmetric decomposition of $J_{1}$ [45] and the $M$ integral (interaction energy integral) [46]. Chang and $\mathrm{Wu}$ [47] proposed the $J_{k}$ method which does not require any auxiliary fields and is suitable for both flat and curved cracks. We note that in the implementation of FEM/XFEM and other domain type methods, these contour integrals are always cast into domain integrals since the FEM solutions and the related quantities (in particular stresses) are known inside the domain [48][49][6]. However, in BEM it is easier to deal with contour integrals, since obtaining solutions inside the domain requires additional integration. While evaluating $J_{k}$ and $M$ integrals along the crack surfaces is done directly and straightforwardly due to the boundary nature of BEM solutions. The latter two contour integral methods, namely $J_{k}$ and $M$ integrals are discussed in detail in this paper.

The isogeometric analysis (IGA) [1] has been proposed as an alternative methodology to the traditional Lagrange polynomial based analyses. The IGA utilizes the same splines, that are used to exactly represent the geometry, as basis functions for the approximation of the unknown fields, which builds up a more direct link between CAD and analysis. Non-uniform rational B-splines (NURBS) based IGA has been widely investigated in many areas [50][51][52][53][54]. 
More flexible geometrical representation techniques, such as T-splines [55][56], PHT splines [57] and LR Splines [58] etc., have been introduced to overcome the major difficulty of NURBS, i.e. the lack of local refinement due to its tensor product structure. Recently the IGA has been incorporated with BEM (namely the isogeometric BEM (IGABEM)) and applied to exterior potential-flow problems [59], potential problems [60], elastostatics [2][3], shape optimization [61], Stokes flow [62] and acoustic [63][64] etc. More recently, IGABEM has been investigated with trimmed NURBS geometry [65][66] and a posteriori error estimator is proposed for adaptive IGABEM [67]. A fast IGABEM solver has been developed in [68].

The IGABEM presents another way for isogeometric analysis due to the natural fit between the two methods. Currently, the dominated CAD geometry only provides surface description by smooth splines. This is in consistence with the basic feature of the BEM since only the unknown fields (displacement and traction) along the boundary is required to approximate. And the convergence of collocation BEM with splines has been investigated which forms a solid basis for the combined methodology [69][70] and latest work can be referred in [71]. In this paper, a new application of IGABEM is discussed in detail for linear elastic fracture problems. It should be noted that knot insertion in B-splines can introduce discontinuities in the geometry, which makes it possible to extend IGA to fracture mechanics [72][10]. The higher order continuity provided by splines also enables a more straightforward expression of the traction BIE for crack modeling. This paper presents a basic scheme for fracture modeling and crack propagation in 2D domains.

The paper is organized as follows: The concept of NURBS is reviewed shortly in section 2 . The basics of the DBEM for fracture modeling are briefly reviewed in section 3, and more details follow, including collocation and singular integration in DBEM. Section 4 details the approaches developed for extraction of the SIFs, based on the $M$ integral and the $J_{k}$ integral. Section 5 outlines a modified NURBS approach to simulate crack growth using NURBS based representation for cracks. Numerical examples are shown both for fracture analysis and crack propagation, in comparison with other popular methods such as SGBEM, XFEM and XEFG. 


\section{NURBS basis functions}

NURBS basis functions are the generalization of B-spline functions that allows a 'projection' from square and cubic domains to form complex geometries. So the basic concept of B-spline is first outlined. B-spline basis functions are defined over a knot vector, which is a non-decreasing sequence of real numbers given in the parameter space. A knot vector is denoted as $\Xi=$ $\left\{\xi_{1}, \xi_{2}, \ldots, \xi_{n+p+1}\right\}$, where $\xi_{A} \in \mathbb{R}$ is the $A^{\text {th }}$ parameter coordinate (knot), $p$ is the order of the polynomial in B-spline basis functions, $n$ is the number of the basis functions. For a given order $p$, the B-spline basis functions $N_{A, p}$ with $1 \leqslant a \leqslant n$ are defined by the Cox-de Boor recursion:

$$
N_{A, 0}(\xi)= \begin{cases}1 & \xi_{A} \leqslant \xi<\xi_{A+1} \\ 0 & \text { otherwise }\end{cases}
$$

then, for $p>0$,

$$
N_{A, p}(\xi)=\frac{\xi-\xi_{A}}{\xi_{A+p}-\xi_{A}} N_{A, p-1}(\xi)+\frac{\xi_{A+p+1}-\xi}{\xi_{A+p+1}-\xi_{A+1}} N_{A+1, p-1}(\xi)
$$

The continuity of B-spline basis functions at $\xi_{A}$ can be decreased by repeating the knot several times. If $\xi_{A}$ has multiplicity $k\left(\xi_{A}=\xi_{A+1}=\ldots=\xi_{A+k-1}\right)$, then the basis functions are $C^{p-k}$ continuous at $\xi_{A}$. Particularly, when $k=p$, the basis is $C^{0}$ and $k=p+1$ leads to a discontinuity at $\xi_{A}$. If the first and last knot have $k=p+1$, the knot vector is called an open knot vector. More details can be referred in [73].

Having defined the B-spline basis functions $\mathbf{N}=\left\{N_{A, p}\right\}_{A=1}^{n}$, we can describe a curve $C(\xi)$ in $\mathbb{R}^{d_{s}}$ ( $d_{s}$ is the spatial dimensionality, $d_{s}=2$ in this paper) by a group of control points $\mathbf{P}=\left\{\mathbf{P}_{A}\right\}_{A=1}^{n}$ with them as:

$$
C(\xi)=\sum_{A=1}^{n} P_{A} N_{A, p}(\xi) .
$$

A NURBS curve is defined in the same way but by replacing the B-spline basis functions by NURBS basis functions. For example, a NURBS curve $C(\xi)$ can be described as:

$$
C(\xi)=\sum_{A=1}^{n} P_{A} R_{A, p}(\xi),
$$




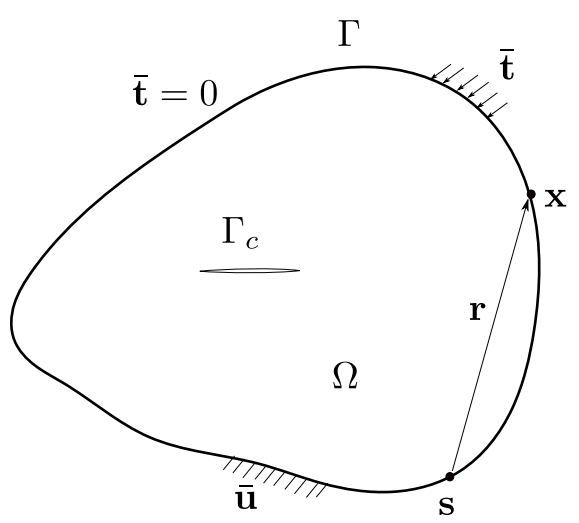

(a) displacement BIE for $\mathbf{s}^{+}$

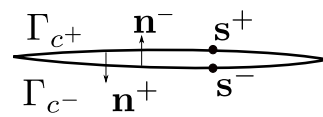

traction BIE for $\mathbf{s}^{-}$

Figure 1: Crack model

where $R_{A, p}$ are the NURBS basis functions, which are defined as

$$
R_{A, p}(\xi)=\frac{\omega_{A} N_{A, p}(\xi)}{\sum_{B=1}^{n} \omega_{B} N_{B, p}(\xi)}
$$

$\omega_{B}$ is the weight associated with the $B^{\text {th }}$ control point. Note that $R_{A, p}$ is only non-zero on the knot interval $\left.\left[\xi_{a}, \xi_{b}\right]\right)$ defined by $p+1$ control points.

\section{$3 \quad$ Isogeometric DBEM for fracture modeling}

\subsection{Problem formulation}

Consider an arbitrary domain $\Omega$ which contains a crack as in Figure 1 . The boundary $\Gamma$ is composed of $\Gamma_{u}$ where Dirichlet boundary conditions are prescribed (known displacement $\overline{\mathbf{u}}$ ), $\Gamma_{t}$ where Neumann boundary conditions are prescribed (known traction $\overline{\mathbf{t}}$ ). The remaining part of the boundary is assumed to be traction free. The crack $\Gamma_{c}$ is composed of two coincident faces: $\Gamma_{c^{+}}$and $\Gamma_{c^{-}}$is assumed also traction free. $\mathbf{s}=\left(s_{1}, s_{2}\right)$ denotes the source point and $\mathbf{x}=\left(x_{1}, x_{2}\right)$ the field point. The displacement BIE at source point $\mathbf{s}$ is given by finding $\mathbf{u}, \mathbf{t}: \Omega \rightarrow \mathbb{R}^{2}$ such that

$$
c_{i j}(\mathbf{s}) u_{j}(\mathbf{s})+f_{\Gamma} T_{i j}(\mathbf{s}, \mathbf{x}) u_{j}(\mathbf{x}) \mathrm{d} \Gamma(\mathbf{x})=\int_{\Gamma} U_{i j}(\mathbf{s}, \mathbf{x}) t_{j}(\mathbf{x}) \mathrm{d} \Gamma(\mathbf{x}),
$$

where the $U_{i j}, T_{i j}$ are called fundamental solutions and for linear elasticity, given by

$$
U_{i j}(\mathbf{s}, \mathbf{x})=\frac{1}{8 \pi \mu(1-\nu)}\left[(3-4 \nu) \delta_{i j} \ln \left(\frac{1}{r}\right)+r_{, i} r_{, j}\right]
$$




$$
T_{i j}(\mathbf{s}, \mathbf{x})=-\frac{1}{4 \pi(1-\nu) r}\left\{\frac{\partial r}{\partial n}\left[(1-2 \nu) \delta_{i j}+2 r_{, i} r_{, j}\right]-(1-2 \nu)\left(r_{, i} n_{j}-r_{, j} n_{i}\right)\right\},
$$

for $2 D$ under plane strain conditions, where $\mu=E /[2(1+\nu)], E$ is Young's Modulus and $\nu$ Poisson's ratio. Components $T_{i j}$ exhibit a singularity of $O(1 / r)$ and the sign $f$ implies that the corresponding integrals are understood in the sense of the Cauchy Principal Value, $|\mathbf{r}|=|\mathbf{x}-\mathbf{s}|$. and $U_{i j}$ is weakly-singular (of order $O(\ln (1 / r))$ ).

The idea of the boundary element method is to discretize the boundary geometry and the physical fields using sets of basis functions. Subsequently, the source point is placed at the collocation points and the displacement BIE (6) is transformed into a corresponding system of linear algebraic equations. However, when the domain contains a crack, the collocation points on the overlapping surfaces (refer to Figure $1(\mathrm{~b})) \Gamma_{c^{+}}$coincide with $\Gamma_{c^{-}}$and the system matrix becomes singular. This difficulty is overcome in dual boundary element methods by prescribing the traction BIE on one of the crack faces $\left(\Gamma_{c^{-}}\right.$in Figure $\left.1(b)\right)$, and the displacement BIE on the other crack surface $\left(\Gamma_{c^{+}}\right)$and on the rest of the boundary $\Gamma$. The traction BIE is obtained by differentiation of the displacement BIE with respect to $\mathbf{s}$ and multiplication by the elastic tensor $E_{i j k l}$ :

$$
\begin{gathered}
c_{i j}(\mathbf{s}) t_{j}(\mathbf{s})+f_{\Gamma} S_{i j}(\mathbf{s}, \mathbf{x}) u_{j}(\mathbf{x}) \mathrm{d} \Gamma(\mathbf{x})=f_{\Gamma} K_{i j}(\mathbf{s}, \mathbf{x}) t_{j}(\mathbf{x}) \mathrm{d} \Gamma(\mathbf{x}), \\
S_{i j}(\mathbf{s}, \mathbf{x})=E_{i k p q} \frac{\partial T_{p j}(\mathbf{s}, \mathbf{x})}{\partial s_{q}} n_{k}(\mathbf{s}), \quad K_{i j}(\mathbf{s}, \mathbf{x})=E_{i k p q} \frac{\partial U_{p j}(\mathbf{s}, \mathbf{x})}{\partial s_{q}} n_{k}(\mathbf{s}),
\end{gathered}
$$

where $S_{i j}$ is the hypersingular kernel $\left(O\left(1 / r^{2}\right)\right)$ and the sign $f$ denotes the Hadamard finite part integrals and $K_{i j}$ is of order $O(1 / r)$. The fundamental solutions for the traction BIE are detailed in Appendix A. $c_{i j}(\mathbf{s})=0.5 \delta_{i j}$ when the source point $\mathbf{s}$ is on a smooth boundary.

\subsection{NURBS discretization of the boundary integral equations}

In the NURBS based isogeometric concept, the physical field is approximated by the same NURBS basis functions as those used to describe the geometry $\Gamma=C(\xi)$. The displacement and traction fields can be approximated as follows:

$$
u_{i}(\xi)=\sum_{A=1}^{n} R_{A, p}(\xi) d_{i}^{A}
$$




$$
t_{i}(\xi)=\sum_{A=1}^{n} R_{A, p}(\xi) q_{i}^{A},
$$

We define an element in the parameter space as an interval between two consecutive nonrepeated knots $\left[\xi_{a}, \xi_{b}\right]$ and linearly map it to interval $[-1,1]$, which is called the parent space [1] and the number of elements is $N_{e}$. We define $\hat{\xi}$ as the parent coordinate of the field point $\mathbf{x}$ in $[-1,1], \hat{\xi}_{s}$ as the parent coordinate of the source point $\mathbf{s}$ in $[-1,1]$, and $J(\hat{\xi})$ is the Jacobian transformation from physical to parent space. The transformation process for one NURBS element (the knot interval $\left[\xi_{a}, \xi_{b}\right]$ ) to the parent space $[-1,1]$ is shown in Figure 2. And we have

$$
\begin{aligned}
\xi=\xi(\hat{\xi}) & =\frac{\left(\xi_{b}-\xi_{a}\right) \hat{\xi}+\left(\xi_{b}+\xi_{a}\right)}{2}, \\
J(\hat{\xi}) & =\frac{\mathrm{d} \Gamma}{\mathrm{d} \xi} \frac{\mathrm{d} \xi}{\mathrm{d} \hat{\xi}}
\end{aligned}
$$

Then the above form can also be written via the elemental approximation as:

$$
\begin{aligned}
& u_{i}(\hat{\xi})=\sum_{I=1}^{p+1} N_{I}(\hat{\xi}) d_{i}^{I}, \\
& t_{i}(\hat{\xi})=\sum_{I=1}^{p+1} N_{I}(\hat{\xi}) q_{i}^{I},
\end{aligned}
$$

where

$$
N_{I}(\hat{\xi})=R_{A, p}(\xi)
$$

And $d_{i}, q_{i}$ are displacement and traction control variables respectively. The relation between the local index $I$ and the global index $A$ is given by the element connectivity [2]. Substituting the discretized displacements and tractions into the BIEs will give,

$$
\begin{aligned}
& \sum_{I=1}^{p+1} C_{i j}^{I}(\mathbf{s}) d_{j}^{I}+\sum_{e=1}^{N_{e}} \sum_{I=1}^{p+1} T_{i j}^{I} d_{j}^{I}=\sum_{e=1}^{N_{e}} \sum_{I=1}^{p+1} U_{i j}^{I} q_{j}^{I}, \\
& \sum_{I=1}^{p+1} C_{i j}^{I}(\mathbf{s}) t_{j}^{I}+\sum_{e=1}^{N_{e}} \sum_{I=1}^{p+1} S_{i j}^{I} d_{j}^{I}=\sum_{e=1}^{N_{e}} \sum_{I=1}^{p+1} K_{i j}^{I} q_{j}^{I},
\end{aligned}
$$

where the jump term and integrals of the fundamental solutions are respectively written as:

$$
C_{i j}^{I}(\mathbf{s})=c_{i j} N_{I}\left(\hat{\xi}_{s}\right)
$$




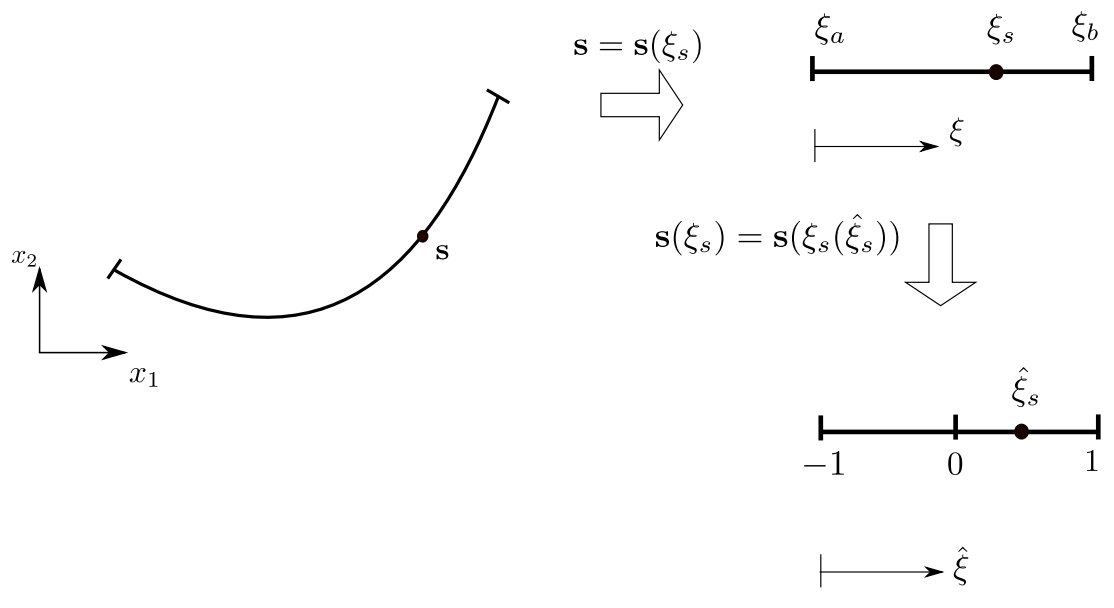

(a)

(b)

Figure 2: Coordinate system in IGABEM: (a) the element containing collocation point $\mathbf{s}$ in the global space; (b)the parametric space and parent space

$$
\begin{aligned}
T_{i j}^{I} & =\int_{-1}^{1} T_{i j}(\mathbf{s}, \mathbf{x}(\hat{\xi})) N_{I}(\hat{\xi}) J(\hat{\xi}) \mathrm{d} \hat{\xi}, \\
U_{i j}^{I} & =\int_{-1}^{1} U_{i j}(\mathbf{s}, \mathbf{x}(\hat{\xi})) N_{I}(\hat{\xi}) J(\hat{\xi}) \mathrm{d} \hat{\xi}, \\
S_{i j}^{I} & =\int_{-1}^{1} S_{i j}(\mathbf{s}, \mathbf{x}(\hat{\xi})) N_{I}(\hat{\xi}) J(\hat{\xi}) \mathrm{d} \hat{\xi}, \\
K_{i j}^{I} & =\int_{-1}^{1} K_{i j}(\mathbf{s}, \mathbf{x}(\hat{\xi})) N_{I}(\hat{\xi}) J(\hat{\xi}) \mathrm{d} \hat{\xi} .
\end{aligned}
$$

\subsection{Treatment of singular integrals}

Integrating the weakly-singular, strongly-singular and hyper-singular kernels in Equations (20)(23) is a major difficulty in BEM. In the present work, weakly-singular integrals are evaluated using Telles' transformation [74]. Strongly-singular integrals in Equation (6) are treated in two different ways. In the first approach, the singularity in $T_{i j}$ is removed by the regularization method, based on use of simple solutions [75][76], i.e. the rigid body motions, which satisfy Equation (6) with zero tractions. Adding and subtracting term $\mathbf{u}(\mathbf{s})$ in Equation (6), the strongly-singular equation can be transformed into the regularized form:

$$
\int_{\Gamma} T_{i j}(\mathbf{s}, \mathbf{x})\left(u_{j}(\mathbf{x})-u_{j}(\mathbf{s})\right) \mathrm{d} \Gamma(\mathbf{x})=\int_{\Gamma} U_{i j}(\mathbf{s}, \mathbf{x}) t_{j}(\mathbf{x}) \mathrm{d} \Gamma(\mathbf{x}) .
$$


After discretization, Equation (24) becomes

$$
\sum_{e=1}^{N_{e}} \sum_{I=1}^{p+1} P_{i j}^{I} d_{j}^{I}=\sum_{e=1}^{N_{e}} \sum_{I=1}^{p+1} U_{i j}^{I} q_{j}^{I},
$$

where

$$
P_{i j}^{I}=\int_{-1}^{1} T_{i j}(\mathbf{s}, \mathbf{x}(\hat{\xi}))\left(N_{I}(\hat{\xi})-N_{I}\left(\hat{\xi}_{s}\right)\right) J(\hat{\xi}) \mathrm{d} \hat{\xi}
$$

The implementation of Equation (24) is simple and does not require calculation of jump term $c_{i j}(\mathbf{s})$. However, when Equation (24) is used at coincident points on crack surfaces, the singularity corresponding to only one of the points is removed. There have been many attempts to overcome this difficulty. For example, creating artificial integration surfaces, excluding the second singular point [77][78] is a possibility. However, the creation and evaluation along the artificial surface is expensive computationally [79] and is particularly cumbersome to deal with in the framework of isogeometric analysis. Therefore, in the present work, Equation (24) is used only on the non-cracked boundary, while on crack surfaces, the approach, known as the singularity subtraction technique (SST), is used [80]. SST is applied to both strongly-singular and hyper-singular integrals after the parametrization in the parent space (Equations (20), (22) and (23)). The essential idea of the method is to expand the production of the kernel function, the shape function and the Jacobian $J(\hat{\xi})$ into Taylor series in the vicinity of the collocation point, and split the integrands into regular and singular parts. Then the singular terms can be evaluated analytically, while for regular terms standard Gauss quadrature is sufficient. Take the hyper-singular integral term $S_{i j l}^{e}$ as an example:

$$
S_{i j}^{I}=\int_{-1}^{1} S_{i j}(\mathbf{s}, \mathbf{x}(\hat{\xi})) N_{I}(\hat{\xi}) J(\hat{\xi}) \mathrm{d} \hat{\xi}=\int_{-1}^{1} F(\hat{\xi}, \hat{\xi}) \mathrm{d} \hat{\xi}
$$

The function $F\left(\hat{\xi}_{s}, \hat{\xi}\right)$ can be expanded as:

$$
F\left(\hat{\xi}_{s}, \hat{\xi}\right)=\frac{F_{-2}\left(\hat{\xi}_{s}\right)}{\delta^{2}}+\frac{F_{-1}\left(\hat{\xi}_{s}\right)}{\delta}+O(1)
$$

where $\delta=\hat{\xi}-\hat{\xi}_{s}$. The details to obtain $F_{-2}$ and $F_{-1}$ with a NURBS basis are given in Appendix 
A and are studied in detail in [80][81]. The final form of (27) is given by:

$$
\begin{aligned}
\int_{-1}^{1} F\left(\hat{\xi_{s}}, \hat{\xi}\right) \mathrm{d} \hat{\xi} & =\int_{-1}^{1}\left(F\left(\hat{\xi_{s}}, \hat{\xi}\right)-\frac{F_{-2}\left(\hat{\xi}_{s}\right)}{\delta^{2}}-\frac{F_{-1}\left(\hat{\xi}_{s}\right)}{\delta}\right) \mathrm{d} \hat{\xi} \\
& +F_{-2}\left(\hat{\xi}_{s}\right)\left(-\frac{1}{1-\hat{\xi}_{s}}+\frac{1}{-1-\hat{\xi}_{s}}\right)+F_{-1}\left(\hat{\xi}_{s}\right) \ln \left|\frac{1-\hat{\xi}_{s}}{-1-\hat{\xi}_{s}}\right|
\end{aligned}
$$

The first integral in (29) is regular and it is evaluated using standard Gaussian quadrature.

\subsection{Partition of unity enrichment formulation}

The partition of unity (PU) enrichment method [82] has been well studied in FEM to model problems with a priori knowledge about the solution. See Sukumar et al [83], Moës et al[84], Gravouil et al [85] for application of XFEM to 3D crack propagation and Bordas and Moran [86], Bordas et al [87], Wyart et al [88] for industrial damage tolerance assessment using XFEM. It was also shown in the literature that the accuracy of the stress intensity factors for 3D linear elastic fracture mechanics was insufficient for coarse meshes and always oscillatory. A posterori error estimate were derived [14][12][11][89] and implemented within the commercial software Morfeo to control the discretization error [12][11].

The approximation of the primary field by PU enrichment is decomposed by two parts: a regular part and an enriched part. The latter allows the approximation to reproduce specific information on the solution through additional degrees of freedom. And the enrichment idea has been introduced within BEM as well [35][63]. Simpson et al [81] first proposed the idea of enrichment in BEM to capture the stress singularity around the crack tip. The enriched displacement approximation with a NURBS basis writes:

$$
u_{i}(\mathbf{x})=\sum_{I \in \mathscr{N}_{I}} N_{I}(\mathbf{x}) d_{i}^{I}+\sum_{J \in \mathscr{N}_{J}} N_{J}(\mathbf{x}) \sum_{l=1}^{4} \phi_{l}(\mathbf{x}) a_{i}^{J},
$$

where $d_{i}^{I}$ are the regular DOFs. $a_{i}^{J}$ are the crack tip enriched DOFs. See [87] for implementation details in an XFEM framework. Since in BEM the crack is explicitly modeled by two overlapping surfaces, the Heaviside enrichment is not required. $\mathscr{N}_{I}$ and $\mathscr{N}_{J}$ are the collections of regular control points and enriched control points, respectively. The crack tip enrichment functions are defined as: 


$$
\left\{\phi_{l}(r, \theta), l=1,4\right\}=\left\{\sqrt{r} \sin \frac{\theta}{2}, \sqrt{r} \cos \frac{\theta}{2}, \sqrt{r} \sin \frac{\theta}{2} \sin \theta, \sqrt{r} \cos \frac{\theta}{2} \sin \theta\right\},
$$

where $(r, \theta)$ are the polar coordinates associated with the crack tip. If the enrichment is done in a small vicinity of the crack tip, where the crack can be regarded as a straight line, i.e. in Equation (31) angle $\theta= \pm \pi$ and the set of four crack tip enrichment functions can be reduced to one, i.e. $\phi(r)=\sqrt{r}$. Then Equation (32) results in:

$$
u_{i}(\mathbf{x})=\sum_{I \in \mathscr{N}_{I}} N_{I}(\mathbf{x}) d_{i}^{I}+\sum_{J \in \mathscr{N}_{J}} N_{J}(\mathbf{x}) \phi(\mathbf{x}) a_{i}^{J}
$$

Substituting the above equation into (6) and (9) and discretizing with a NURBS basis, the enriched displacement and traction boundary integral equations can be obtained, respectively:

$$
\begin{gathered}
\sum_{I}^{p+1} C_{i j}^{I}(\mathbf{s})\left(d_{j}^{I}+\phi(\mathbf{s}) a_{j}^{I}\right)+\sum_{e=1}^{N_{e}} \sum_{I}^{p+1}\left(T_{i j}^{I} d_{j}^{I}+T_{i j}^{I} \phi a_{j}^{I}\right)=\sum_{e=1}^{N_{e}} \sum_{I}^{p+1} U_{i j}^{I} q_{j}^{I}, \\
\sum_{I}^{p+1} C_{i j}^{I}(\mathbf{s}) t_{j}^{I}+\sum_{e=1}^{N_{e}} \sum_{I}^{p+1}\left(S_{i j}^{I} d_{j}^{I}+S_{i j}^{I} \phi a_{j}^{I}\right)=\sum_{e=1}^{N_{e}} \sum_{I}^{p+1} K_{i j}^{I} q_{j}^{I} .
\end{gathered}
$$

Note that topological enrichment is used, i.e. only the elements containing the crack tip are enriched, the enrichment terms do not need to be computed for unenriched elements. Differing from [81] where the discontinuous quadratic Lagrange elements are enriched, the enrichment for the NURBS basis will lead to blending elements due to the continuity of the basis. The singular integration for enriched elements can be done with SST as in section 3.3 as long as the local expansion for $\phi(r)=\sqrt{r}$ at the collocation point with respect to intrinsic coordinate is written explicitly.

\subsection{Continuity requirements and collocation strategy}

Methods for evaluating strongly-singular and hyper-singular integrals (20), (22), (23), described above, are implicitly or explicitly based on Taylor expansions of the integrands in the vicinity of the collocation point. Since the essential feature of the isogemetric approach is to represent displacements, tractions and the geometry using the same NURBS basis functions, special attention should be paid to the continuity of NURBS basis functions at the collocation points 


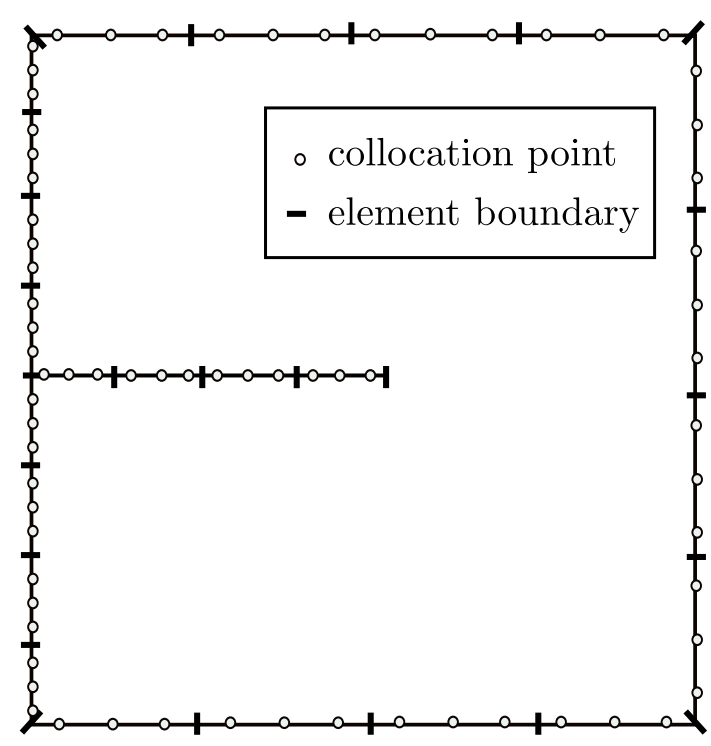

(a)

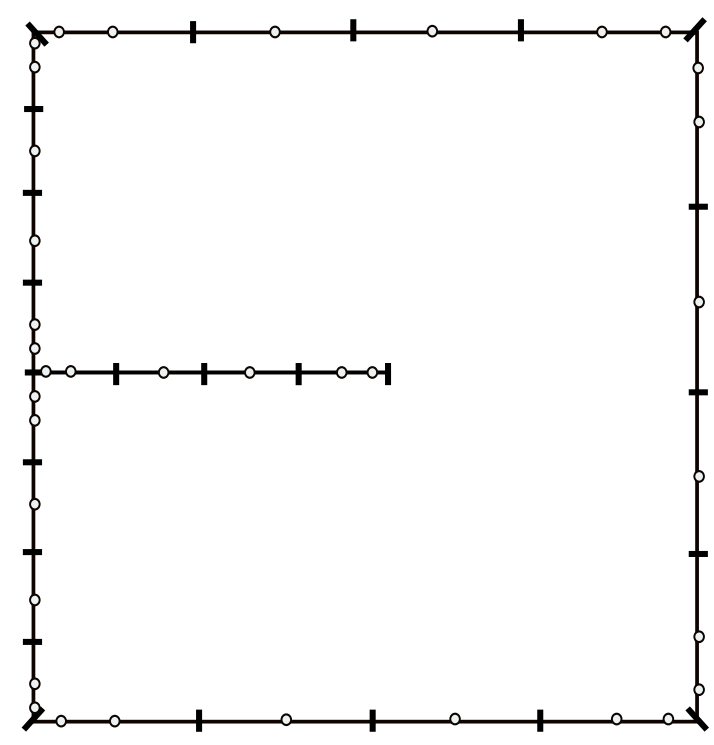

(b)

Figure 3: Mesh discretization for a mode I crack: (a) discontinuous Lagrange element $(p=2)$, (b) NURBS $(p=2)$

where the Taylor series are expanded.

In the classical boundary element method a common way to guarantee the existence of integrals in (20), (22), (23) is by the so-called discontinuous quadratic Largange elements [22], i.e. placing collocation points inside an element, where the quadratic polynomials are $C^{\infty}$ continuous. The same approach can be implemented with NURBS parametrization, since inside the elements NURBS basis functions are infinitely smooth, i.e. the SST can be used directly to treat all singularities. In Figure 3 (a) and (b) examples of boundary discretization are shown for classical BEM and IGABEM respectively, where the collocation points in IGA are generated by Greville abscissae [90] and the collocation points are moved inside the elements when higher order continuity is necessary.

For the enrichment formulation, since enriched DOFs are introduced, additional source points need to be collocated to balance the number of system unknowns. The location of the source points plays an important role in the condition number of the BEM system matrix. It reveals that for crack tip enrichment, when the additional collocation points are inside the enriched element, the system condition remains small and gives accurate solutions (see [81] for more details). Nevertheless, the specific location inside the crack tip element has little influence on 

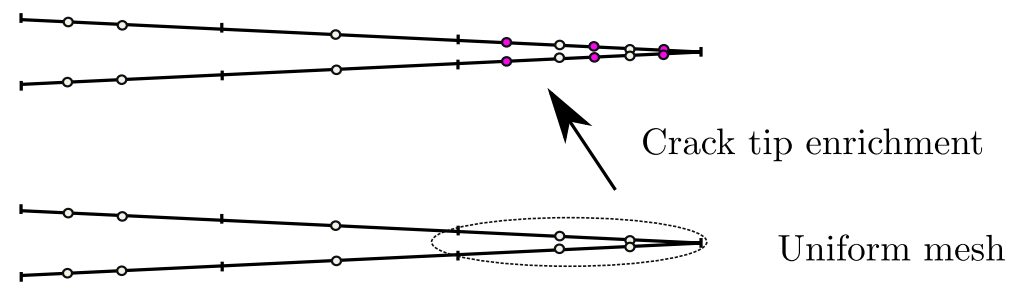

- Original collocation point

- Addtional collocation point
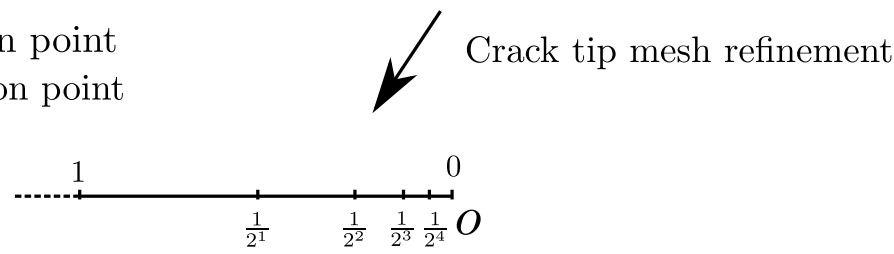

Figure 4: Mesh and collocation for crack surfaces

the final results. Hence in this work, the additional source points are inserted within the crack tip element and spread uniformly between the original collocation points. Figure (4) illustrates the scheme applied in this paper for collocation on the crack surface.

However, the classical theory of boundary integral equations admits much weaker continuity requirements, i.e. the Cauchy and Hadamard integrals exist for $C^{1, \alpha}(\Gamma)(0<\alpha<1)$ density functions (known as Hölder continuous) [91]. Therefore, strongly singular and hyper-singular equations, and all the more so the regularized equation (24), can be used at collocation points located at the edges of the elements in IGABEM, provided that the NURBS basis is sufficiently smooth. However, optimal collocation strategies remain the subject of further research, and require more detailed theoretical and numerical studies.

\section{Evaluation of stress intensity factors}

\section{$4.1 \quad J_{k}$-integral}

In this section, two different kinds of $J$ integral based methods for the extraction of SIFs are briefly reviewed. The first one is the $J_{k}$ method proposed in [47], which is the more general case of the $\mathrm{J}$ integral. The definition of the $J_{k}$ in $2 \mathrm{D}$ is given as:

$$
J_{k}:=\lim _{\Gamma_{\epsilon} \rightarrow 0} \int_{\Gamma_{\epsilon}}\left(W \delta_{j k}-\sigma_{i j} u_{i, k}\right) n_{j} \mathrm{~d} \Gamma=\lim _{\Gamma_{\epsilon} \rightarrow 0} \int_{\Gamma_{\epsilon}} P_{k j} n_{j} \mathrm{~d} \Gamma
$$

where $P_{k j}$ is the Eshelby tensor, $W=1 / 2 \sigma_{i j} \epsilon_{i j}$ is the strain energy density, $n_{j}$ is the unit outward normal of $\Gamma_{\epsilon}$. $J_{1}$ represents a special case, the $J$ integral. Throughout the paper we 


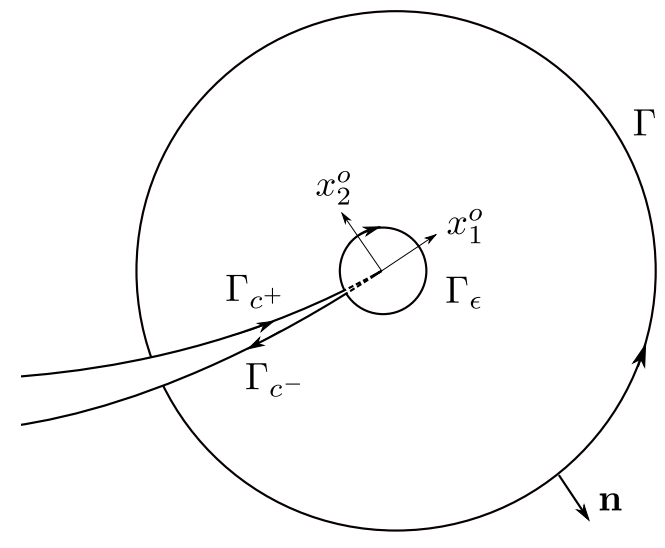

(a)

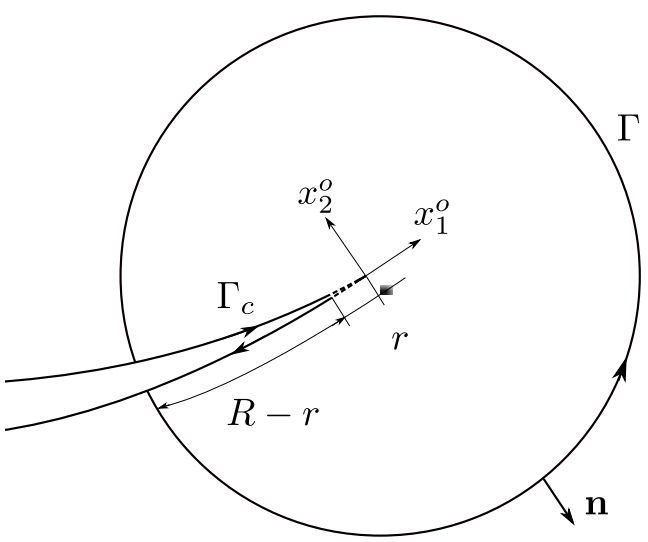

(b)

Figure 5: Path definition for $J$ integral

will use these two notations interchangeably. All the variables are defined in the crack tip local coordinate system $\left(x_{0}, y_{0}\right)$ as in Figure 5 (a). However, from the numerical point of view, it is difficult to calculate the limit in Equation (35), so that the definition of $J_{k}$ is usually modified in the following way. Since the integral of the Eshelby tensor is equal to zero for any closed contour, which does not contain a defect, additional contours $\Gamma, \Gamma_{c^{+}}, \Gamma_{c^{-}}$are introduced, such that Equation (35) can be rewritten as [92]

$$
J_{k}=\lim _{\Gamma_{\epsilon} \rightarrow 0} \int_{\Gamma_{\epsilon}} P_{k j} n_{j} \mathrm{~d} \Gamma=\int_{\Gamma} P_{k j} n_{j} \mathrm{~d} \Gamma+\int_{\Gamma_{c^{+}}} P_{k j} n_{j} \mathrm{~d} \Gamma+\int_{\Gamma_{c^{-}}} P_{k j} n_{j} \mathrm{~d} \Gamma
$$

When $k=1$, for a flat crack $n_{1}=0$ along the crack surfaces and thus along the contours $\Gamma_{c^{+}}$ and $\Gamma_{c^{-}}$the integral is zero, and Equation (36) simplifies to:

$$
J_{1}=\int_{\Gamma} P_{1 j} n_{j} \mathrm{~d} \Gamma
$$

This expression shows the path independence of the $J$ integral for a flat crack. But for the $J_{2}$ integral, the term associated with the crack surface cannot be omitted since $n_{2}=1$ and this term leads to a singularity in numerical evaluation.

The most general 2D scenario must account for curved cracks. The associated contribution from the crack surfaces to both $J_{1}$ and $J_{2}$ cannot in general be neglected. It should be noted that the energy density $W \rightarrow 1 / r$ when approaching the crack tip since both $\sigma_{i j}$ and $\epsilon_{i j}$ tend to $1 / \sqrt{r}$. The integrand along the crack surface will remain of $O(1 / r)$, and this kind of singular 
integral cannot be treated in a regular way. In [92] and [47], the crack surface was split into a far field part and a near-tip part (Figure 5(b)) in order to evaluate the singular integral:

$$
J_{k}=\int_{\Gamma} P_{k j} n_{j} \mathrm{~d} \Gamma+\int_{R-r} \llbracket W \rrbracket n_{k}^{+} \mathrm{d} \Gamma+\int_{r} \llbracket W \rrbracket n_{k}^{+} \mathrm{d} \Gamma
$$

The far field part is integrated by regular Gauss quadrature. The near-tip part integral on the crack surface can be simply omitted for $J_{1}(k=1)$, since $n_{1}$ is mostly zero, while for $J_{2}(k=2)$, the near-tip part exhibits the $O(1 / r)$ singularity. The energy jump $\llbracket W \rrbracket$ on the near-tip surface can be evaluated as in [92]:

$$
\llbracket W \rrbracket=\frac{-4 K_{I I} \sigma_{x 0}}{E \sqrt{2 \pi r}}+O\left(r^{1 / 2}\right)
$$

where $\sigma_{x 0}$ is called T-stress. Thus near-tip part of $\llbracket W \rrbracket$ can be represented as a proportion to the $r^{1 / 2}$

$$
J_{k}=\int_{\Gamma} P_{k j} n_{j} \mathrm{~d} \Gamma+\int_{R-r} \llbracket W \rrbracket n_{k}^{+} \mathrm{d} \Gamma+\Lambda n_{k} r^{1 / 2}
$$

Since two unknown variables $J_{2}$ and $\Lambda$ appear in the above equation, the integral cannot be evaluated at once. The splitting procedure needs to be performed several times by taking different $r$, and a group of values of $J_{2}$ and $\Lambda$ can be found in order to extrapolate $J_{2}$ for the case of no splitting. In Equation (40), as long as the $O\left(1 / r^{1 / 2}\right)$ can be captured, the $J_{k}$ integral can be correctly evaluated and the SIFs can be deduced (see Appendix B). Nevertheless, the choice of the extraction radius ' $r$ ' becomes path dependent and problem dependent in real applications.

\section{2 $M$ integral}

The $M$ integral is another possible method to extract the SIFs. By applying the $J$ integral under two states, the actual state (denoted with superscript ' 1 '), and the auxiliary state (superscript '2'), and adding them together:

$$
J^{(1+2)}=\int_{\Gamma_{\epsilon}}\left[\frac{1}{2}\left(\sigma_{i j}^{(1)}+\sigma_{i j}^{(2)}\right)\left(\epsilon_{i j}^{(1)}+\epsilon_{i j}^{(2)}\right) \delta_{1 j}-\left(\sigma_{i j}^{(1)}+\sigma_{i j}^{(2)}\right) \frac{\partial\left(u_{i}^{(1)}+u_{i}^{(2)}\right)}{\partial x_{1}}\right] n_{j} \mathrm{~d} \Gamma .
$$

Rearranging the two state terms gives

$$
J^{(1+2)}=J^{(1)}+J^{(2)}+M^{(1,2)},
$$


where

$$
\begin{gathered}
M^{(1,2)}=\int_{\Gamma_{\epsilon}}\left[W^{(1,2)} \delta_{1 j}-\sigma_{i j}^{(1)} \frac{\partial u_{i}^{(2)}}{\partial x_{1}}-\sigma_{i j}^{(2)} \frac{\partial u_{i}^{(1)}}{\partial x_{1}}\right] n_{j} \mathrm{~d} \Gamma \\
W^{(1,2)}=\sigma_{i j}^{(1)} \epsilon_{i j}^{(2)}=\sigma_{i j}^{(2)} \epsilon_{i j}^{(1)}
\end{gathered}
$$

Once the $M$ integral has been evaluated, the SIFs can be extracted directly (see Appendix B). But we note that in Yau et al's work [46], a flat crack surface is assumed. When applied to practical problems, the radius of the contour circle should be chosen 'small enough' to guarantee that within the domain bounded by $\Gamma$, the crack is 'almost' straight.

In this paper, the $M$ integral is adopted. A detailed comparison of both methods applied to curved cracks is provided in the forthcoming sections.

Once the SIFs have been obtained, the maximum hoop stress criterion is used to determine the direction of crack propagation. We assume that the crack propagates in the direction $\theta_{c}$ such that the hoop stress is maximum, which is given (see [93], for example) by the following expression. Note that the quantity of interest determining the accuracy of each propagation step is the ratio $\left(K_{I I} / K_{I}\right)$

$$
\theta_{c}=2 \arctan \left[\frac{-2\left(K_{I I} / K_{I}\right)}{1+\sqrt{1+8\left(K_{I I} / K_{I}\right)^{2}}}\right]
$$

\section{$5 \quad 2 \mathrm{D}$ NURBS crack propagation}

A NURBS crack propagation algorithm is outlined next. The conceptual idea for the deformation of the NURBS curve representing the crack is realised by moving the control points to make the curve satisfy the external constraints under a user-defined function [94]. For crack growth, the external constraint is the movement of the position of crack tip (or crack front in 3D). Paluszny et al implemented the idea in FEM to represent crack growth or intersection by updating the control points to satisfy the constraints given by fracture parameters [95]. The algorithm is briefly reviewed as follows:

- Initiation: represent the crack by the NURBS curve;

- Calculate the new physical position of the crack tip $M^{\prime}$ (the space constraint). This is determined by specified fracture criterion given in section 4.2 ; 


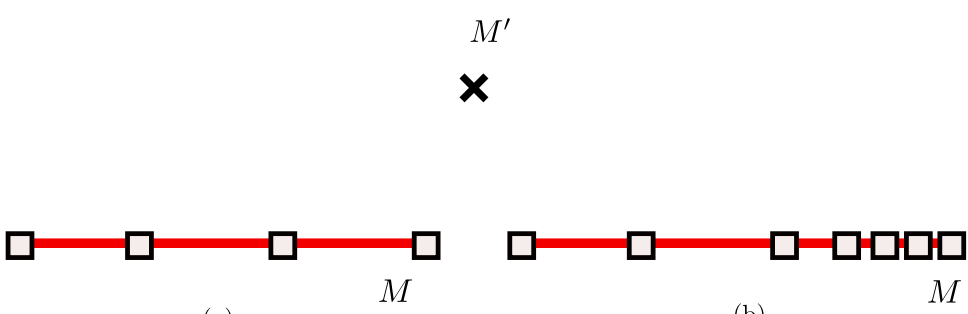

(a)

(b)

\section{$M^{\prime}$}

$\mathbf{x}$

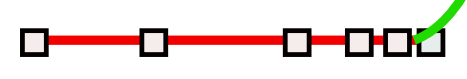

(c)

Figure 6: NURBS modification for crack growth. (a)Original crack and new crack tip $M^{\prime}$; (b)Knot insertion to refine the crack tip element; (c)Move the control points to obtain new crack curve by the presented algorithm

- Specify the parametric coordinate $\xi$ (the parametric constraint) of the old crack tip $M$;

- Define the influence functions $f$. Here for $2 \mathrm{D}$ fracture these functions are selected as the NURBS basis functions at the parametric constraint $\xi$ (which is called natural deformation in [94]). $f(A)=R_{A, p}(\xi), A=1, \ldots, n, n$ is the number of NURBS basis function of the corresponding control point $P_{A}$.

- Calculating the motion vector of each control point $\mathbf{m}(A)$ : the movement of the control points is given by

$$
\mathbf{m}(A)=\frac{f(A)}{\sum_{B=1}^{n} R_{B, p}(\xi) f(B)} \mathbf{e}, \quad \mathbf{e}=\overrightarrow{M M^{\prime}}
$$

The process to stretch a NURBS curve to simulate crack growth in 2D is illustrated in Figure

6. Certain knot insertion should be done at the crack tip element in order to capture the local changes. We note that refining the crack tip element also helps improve the solution near the crack tip, and a graded mesh refinement is designed as in Figure 4, where the new knots are inserted consecutively at the $(1 / 2)^{i}, \quad i=1,2,3,4 \ldots$ of the distance to the crack tip in parametric space (the obtained meshes are denoted as $R 1, R 2, R 3, R 4 \ldots$ ).

\section{$6 \quad$ Numerical examples}

In this section, several numerical examples are presented to verify the proposed method for fracture analysis. We first give examples to study the behavior of the (X)IGABEM on static fracture analysis. Then the application to crack propagation by comparing against XFEM is demonstrated. A fixed number of Gauss points $(n g p=30)$ is adopted for the integration of both singular and nearly-singular integrals, although we note that it would be desirable to develop 


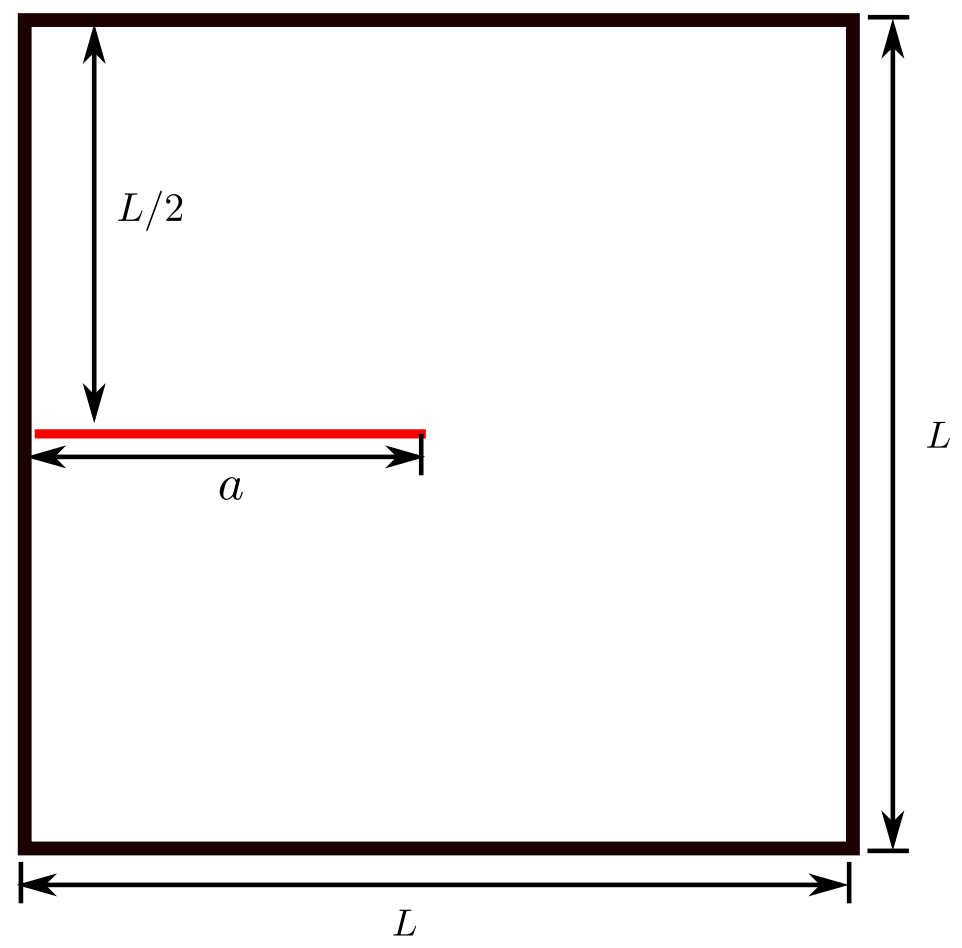

Figure 7: Edge crack

adaptive quadrature rules for the nearly-singular integrals in BEM. The order of NURBS basis and discontinuous lagrange basis is taken as 2 for all the examples.

\subsection{Edge crack}

Figure 7 illustrates the chosen edge crack problem. we use the first-term asymptotic solution of a crack problem [96] (refer to the auxiliary displacements in Appendix B), which we prescribe as Dirichlet boundary condition on the outer boundaries, while keeping crack faces traction free. The parameters $E=1, \nu=0.3, a=1, L=2$. For a mode I crack, $K_{I}=1, K_{I I}=0$ and for a mode II crack, $K_{I}=0, K_{I I}=1$. Thus the numerical displacement field on the crack as well as the SIFs can be compared to the analytical solution.

\subsubsection{Ability of the method to capture the crack tip singularity}

An accurate approximation of the solution near the crack tip is crucial to the accurate evaluation of fracture parameters such as the SIFs. Three scenarios are studied here, uniform meshes, graded refinement and enrichment of the crack tip element with function given in Equation (32). Figure 8 shows the displacement $u_{y}$ along the upper crack surface for the mode I problem. 


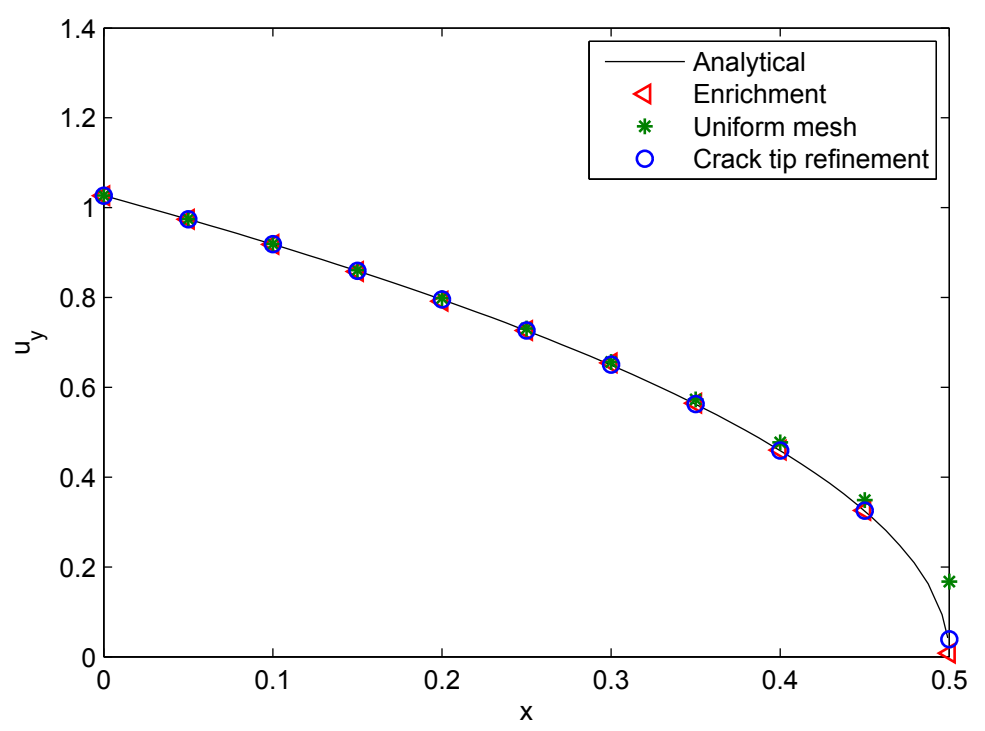

Figure 8: $u_{y}$ along the upper crack surface. It can be observed that in all cases, the numerical displacements agree well with the analytical solution, even for coarse meshes. The graded refinement and enrichment method both give improved results near the crack tip

The crack is discretized by 3 uniform elements. It can be observed that in all cases, the numerical displacements agree well with the analytical solution, even for coarse meshes. The graded refinement and enrichment method both give improved results near the crack tip. To further assess the accuracy of these methods, the error in the displacement $L_{2}$ norm of the displacements along the crack surfaces, given by

$$
e_{L_{2}}=\sqrt{\frac{\int_{\Gamma_{c}}\left(\mathbf{u}-\mathbf{u}_{e x t}\right)^{\mathrm{T}}\left(\mathbf{u}-\mathbf{u}_{e x t}\right) \mathrm{d} \Gamma}{\int_{\Gamma_{c}} \mathbf{u}_{e x t}^{\mathrm{T}} \mathbf{u}_{e x t} \mathrm{~d} \Gamma}}
$$

is plotted in Figure 9. We check the convergence results by inserting the knots at $(1 / 2)^{i}$ consecutively until $i=4$ described in Figure 4 (the results are denoted as $R 1, R 2, R 3$ and $R 4$ respectively). It can be seen that enrichment achieves an accuracy which is intermediate between $R 3$ and $R 4$ graded meshes while the convergence rate is improved by $55 \%$ compared to the graded mesh refinement. In the following examples for static crack and crack propagation, the graded mesh refinement by 4 successive knot insertions is used for study further. 


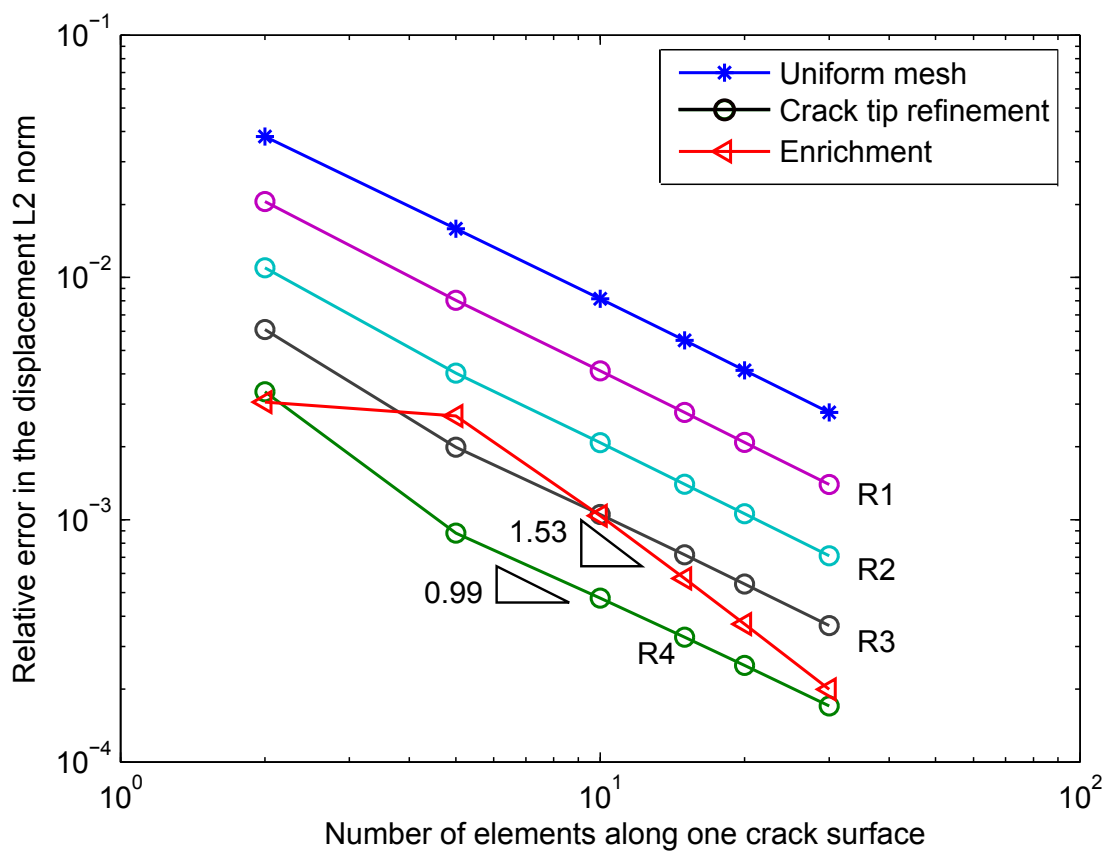

Figure 9: Relative error in $L_{2}$ norm of the displacement along the crack surface. Note that $R 1$, $R 2, R 3$ and $R 4$ correspond to the crack tip element's refinement by consecutive knot insertions at $(1 / 2)^{1},(1 / 2)^{2},(1 / 2)^{3}$ and $(1 / 2)^{4}$ of the distance to the crack tip in parametric space. It can be seen that enrichment achieves an accuracy which is intermediate between $R 3$ and $R 4$ graded meshes while the convergence rate is improved by $55 \%$ compared to the graded mesh refinement

\subsubsection{SIFs comparison with Lagrange basis}

To evaluate the potential of IGABEM for fracture, the SIFs given by the $M$ integral are compared to those from Lagrange elements using uniform meshes and no special treatment for the crack tip. The radius for the $M$ integral is taken as the distance from the crack tip to the third collocation point counting from the crack tip, thus with mesh refinement, the extraction domain will shrink. A convergence check for the error in the normalized SIFs $K_{I}, K_{I I}$ is shown in Figure 10. It can be observed that the precision provided by NURBS basis is much higher (one order of magnitude for approximately 500 DOFs) than that of discontinuous Lagrange basis. Because discontinuous Lagrange basis typically leads to more nodes than NURBS basis for a given number of elements (as presented in Figure 3), the convergence results are re-plotted in terms of number of elements in Figure 11. For the two coarsest meshes of 4 elements per edge, the Lagrange basis is more accurate than NURBS, but with mesh refinement, the NURBS becomes superior, due to a larger convergence rate. From both figures, it is observed that the convergence rates of SIFs by NURBS basis is $5 \sim 8$ times higher than those by discontinuous 


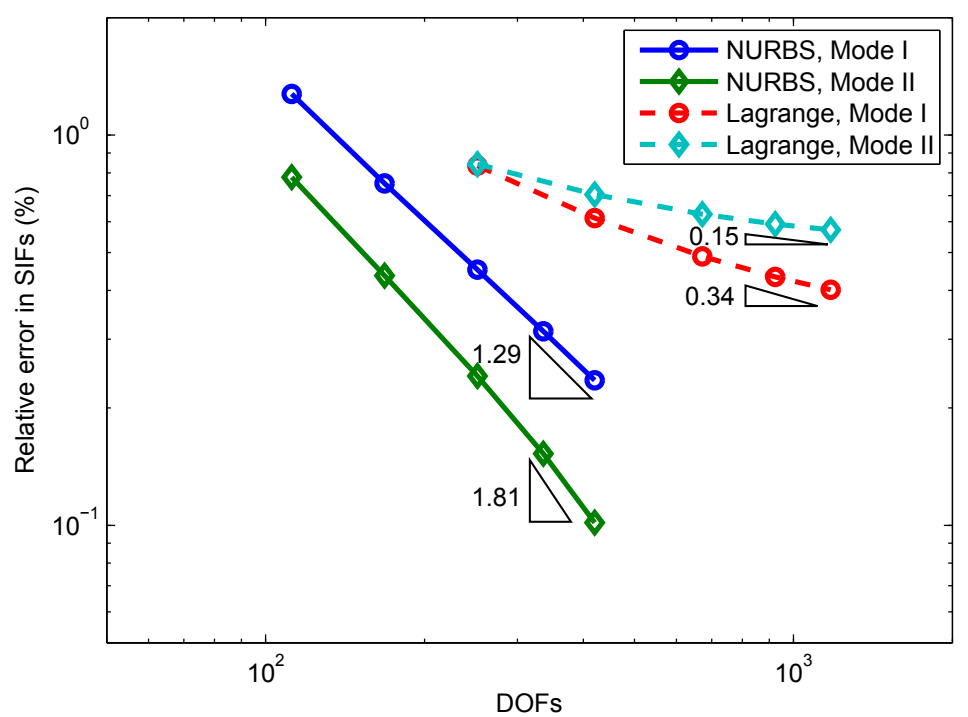

Figure 10: Convergence results of SIF for the mode I and mode II crack. It can be observed that the precision provided by NURBS basis is much higher (one order of magnitude for approximately 500 DOFs) than that of discontinuous Lagrange basis

Lagrange basis.

\subsection{Inclined centre crack}

In this example, The SIFs are calculated for a plate with an inclined crack under remote biaxial tension such that $\sigma=\sigma_{0}$ is applied in the $y$-direction and $\sigma=\lambda \sigma_{0}$ is applied in the $x$-direction, where $\lambda$ is the load ratio and $\sigma_{0}=1$. The inclined centre crack with angle $\beta$ varies from 0 to $\pi / 2$, see Figure 12. The edge length of the plate $L=1$, crack length $2 a=0.02 . L>>a$ so that the numerical results can be compared with the analytical solution for an infinite plate, given in [97]. The elasticity parameters are $E=1, \nu=0.3$. The SIFs in this example obtained by the $M$ integral can be compared to the analytical ones as follows:

$$
\begin{aligned}
& K_{I}=\sigma \sqrt{\pi a}\left(\cos ^{2} \beta+\lambda \sin ^{2} \beta\right), \\
& K_{I I}=\sigma \sqrt{\pi a}(1-\lambda) \cos \beta \sin \beta .
\end{aligned}
$$

The mesh of the crack surface was refined uniformly for both the discontinuous Lagrange basis BEM (LBEM) and NURBS (IGABEM). The local graded refinement for crack tip elements described in Figure 4 is also performed (the corresponding result is denoted as IGABEM(r)). 


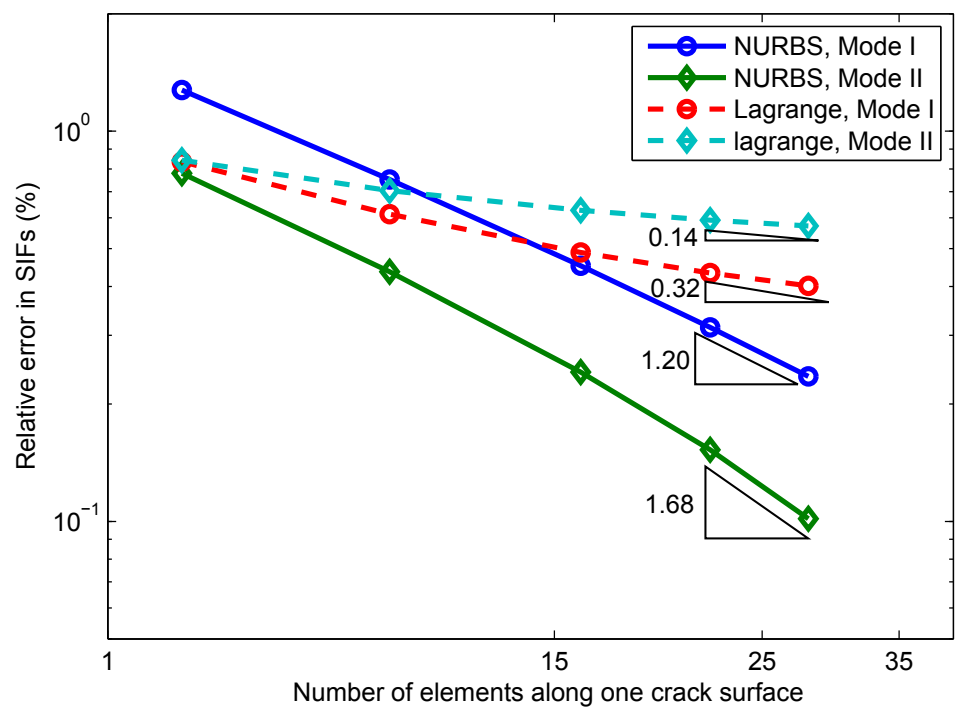

Figure 11: Convergence results of SIF for the mode I and mode II crack, plotting in terms of element number

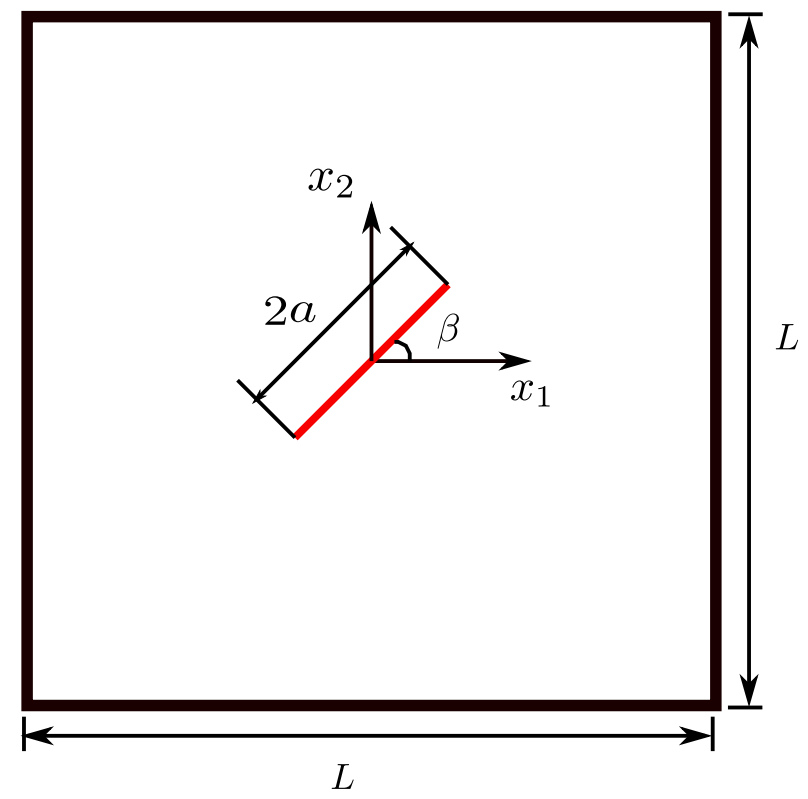

Figure 12: Physical model of an inclined center crack problem 
Assuming the number of elements for the crack is $m$, a convergence check is done with the crack angle $\beta=\pi / 6$ at load ratio $\lambda=0.5$ (biaxially loaded). The results are given in Table 2 and 3. Here the SGBEM results [30] are also given as a reference. It can be concluded that the proposed local crack tip refinement gives a very good accuracy for practical applications.

The SIFs are then compared for different angles at $\lambda=0$ (uniaxially loaded). In this case, the crack is discretized by 4 uniform elements, and for IGABEM, the crack tip element is further refined in the same fashion. The SIFs are given in Table 4.

\begin{tabular}{|c|c|c|c|c|}
\hline & \multicolumn{4}{|c|}{$K_{I} / K_{I}^{\text {exact }}$} \\
\hline$m$ & SGBEM & LBEM & IGABEM & IGABEM(r) \\
\hline 3 & 0.9913 & 1.00451 & 1.00982 & 1.00120 \\
\hline 4 & 1.0002 & 1.00333 & 1.00769 & 1.00105 \\
\hline 5 & 1.0001 & 1.00268 & 1.00633 & 1.00090 \\
\hline 6 & 1.0002 & 1.00230 & 1.00539 & 1.00080 \\
\hline 7 & 1.0003 & 1.00206 & 1.00474 & 1.00074 \\
\hline 8 & 1.0003 & 1.00190 & 1.00426 & 1.00070 \\
\hline 9 & 1.0003 & 1.00177 & 1.00389 & 1.00066 \\
\hline 10 & 1.0003 & 1.00167 & 1.00359 & 1.00064 \\
\hline 11 & 1.0003 & 1.00159 & 1.00336 & 1.00062 \\
\hline 12 & 1.0003 & 1.00152 & 1.00316 & 1.00060 \\
\hline 14 & 1.0003 & 1.00142 & 1.00285 & 1.00058 \\
\hline
\end{tabular}

Table 2: Normalized $K_{I}$ in inclined centre crack

\begin{tabular}{|c|c|c|c|c|}
\hline & \multicolumn{5}{|c|}{$K_{I I} / K_{I I}^{\text {exact }}$} \\
\hline$m$ & SGBEM & LBEM & IGABEM & IGABEM(r) \\
\hline 3 & 1.0075 & 1.00104 & 1.00647 & 1.00146 \\
\hline 4 & 1.0009 & 1.00129 & 1.00656 & 1.00129 \\
\hline 5 & 1.0010 & 1.00158 & 1.00607 & 1.00113 \\
\hline 6 & 1.0009 & 1.00160 & 1.00550 & 1.00102 \\
\hline 7 & 1.0014 & 1.00153 & 1.00500 & 1.00096 \\
\hline 8 & 1.0005 & 1.00143 & 1.00458 & 1.00091 \\
\hline 9 & 0.9997 & 1.00134 & 1.00424 & 1.00087 \\
\hline 10 & 1.0009 & 1.00126 & 1.00396 & 1.00085 \\
\hline 11 & 0.9992 & 1.00119 & 1.00373 & 1.00083 \\
\hline 12 & 1.0013 & 1.00112 & 1.00353 & 1.00081 \\
\hline 14 & 1.0004 & 1.00102 & 1.00322 & 1.00079 \\
\hline
\end{tabular}

Table 3: Normalized $K_{I I}$ in inclined centre crack

\subsection{Arc crack}

The circular arc crack under remote uniform biaxial tension is used to further validate the effectiveness of the proposed method for curved cracks. The problem is defined in Figure 13. 


\begin{tabular}{|l|l|l|l|l|l|l|}
\hline & \multicolumn{3}{|c|}{$K_{I}$} & \multicolumn{3}{c|}{$K_{I I}$} \\
\hline$\beta$ & Exact & IGABEM $(\mathrm{r})$ & SGBEM & Exact & IGABEM(r) & SGBEM \\
\hline 0 & 1.0000 & $1.0006(6.0 e-4)$ & $1.0002(2.0 e-4)$ & 0.0000 & $0.0000(<1 . e-4)$ & $0.0000(<1 . e-4)$ \\
\hline$\pi / 12$ & 0.9330 & $0.9336(6.4 e-4)$ & $0.9332(2.1 e-4)$ & 0.2500 & $0.2503(1.2 e-3)$ & $0.2502(8.0 e-4)$ \\
\hline$\pi / 6$ & 0.7500 & $0.7505(6.7 e-4)$ & $0.7502(2.7 e-4)$ & 0.4330 & $0.4336(1.4 e-3)$ & $0.4334(9.2 e-4)$ \\
\hline$\pi / 4$ & 0.5000 & $0.5003(6.0 e-4)$ & $0.5001(2.0 e-4)$ & 0.5000 & $0.5006(1.2 e-3)$ & $0.5004(6.0 e-4)$ \\
\hline$\pi / 3$ & 0.2500 & $0.2501(4.0 e-4)$ & $0.2500(<1 . e-4)$ & 0.4330 & $0.4335(1.2 e-3)$ & $0.4333(6.9 e-4)$ \\
\hline $5 \pi / 12$ & 0.0670 & $0.0670(<1 . e-4)$ & $0.0670(<1 . e-4)$ & 0.2500 & $0.2503(1.2 e-3)$ & $0.2502(8.0 e-4)$ \\
\hline$\pi / 2$ & 0.0000 & $0.0000(<1 . e-4)$ & $0.0000(<1 . e-4)$ & 0.0000 & $0.0000(<1 . e-4)$ & $0.0000(<1 . e-4)$ \\
\hline
\end{tabular}

Table 4: SIFs and relative error (in brackets) for the inclined centre crack

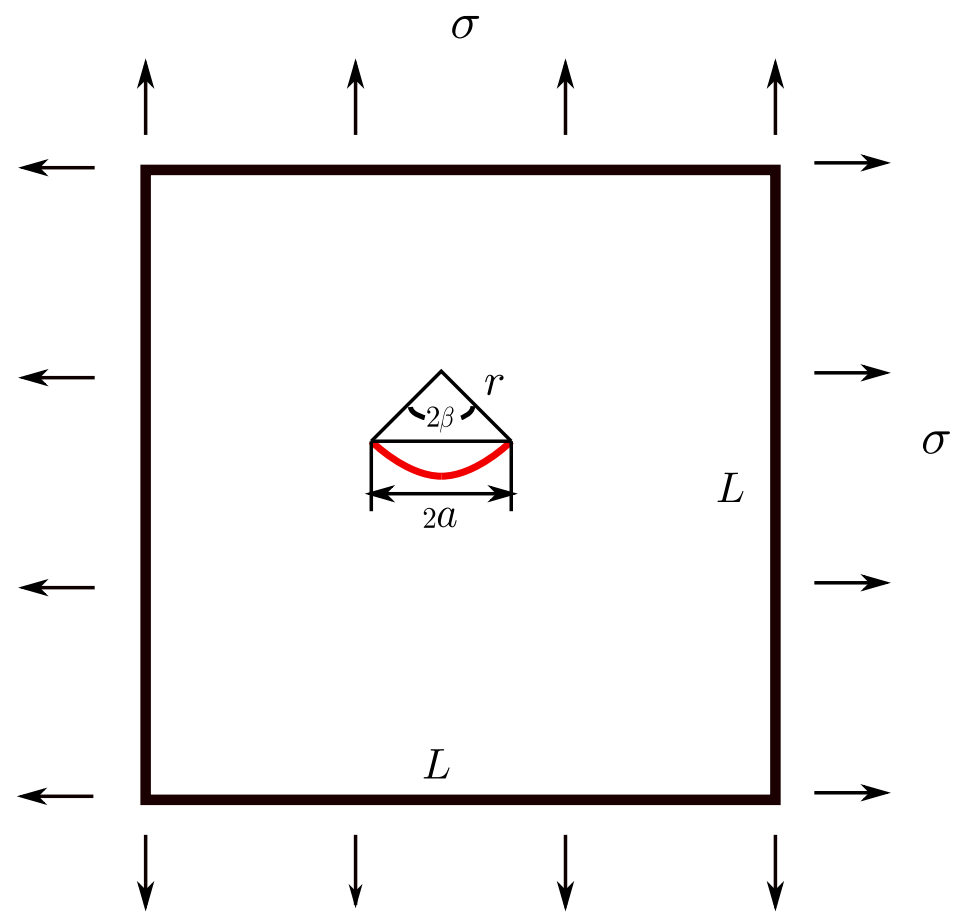

Figure 13: Physical model of the arc crack 
Here $L=1,2 a=0.01, L>>a, E=1, \nu=0.3$. In the test $\sigma=1, \beta=\pi / 4$. The analytical SIFs are given by [98] as:

$$
\begin{aligned}
K_{I} & =\sigma \sqrt{\pi a} \frac{\cos (\beta / 2)}{1+\sin ^{2}(\beta / 2)}, \\
K_{I I} & =\sigma \sqrt{\pi a} \frac{\sin (\beta / 2)}{1+\sin ^{2}(\beta / 2)} .
\end{aligned}
$$

$m$ elements are used to discretize each crack surface with crack tip elements refined as in Figure 4. A convergence check for the SIFs are listed in Table 5. Here the SIF extraction from both the $J_{k}$ integral method and the $M$ integral method are compared. Both methods use the same radius $R$, and the partition of the crack surface for the $J_{k}$ integral is done by experience at $r=0.03 R, 0.04 R, 0.05 R, 0.06 R, 0.07 R$. The results of the two methods are comparable, differing only at the fourth digit. But we note that the $J_{k}$ integral method is more computationally expensive than the $M$ integral as (1) it needs integration on the crack surfaces; (2) the crack surface needs to be partitioned into two parts; (3) the integration needs to be performed several times as described in section 4.1 .

\begin{tabular}{|c|c|c|c|c|}
\hline & \multicolumn{2}{|c|}{$K_{I} / K_{I}^{\text {exact }}$} & \multicolumn{2}{c|}{$K_{I I} / K_{I I}^{\text {exact }}$} \\
\hline$m$ & $M$ integral & $J_{k}$ integral & $M$ integral & $J_{k}$ integral \\
\hline 10 & 1.00045 & 0.99972 & 0.97506 & 1.00309 \\
\hline 14 & 1.00014 & 0.99979 & 0.98621 & 1.00248 \\
\hline 17 & 1.00011 & 0.99982 & 0.98642 & 1.00217 \\
\hline 20 & 1.00009 & 0.99985 & 0.98657 & 1.00195 \\
\hline 23 & 1.00002 & 0.99987 & 0.99407 & 1.00176 \\
\hline 26 & 1.00002 & 0.99989 & 0.99413 & 1.00163 \\
\hline
\end{tabular}

Table 5: SIFs for the arc crack

\subsection{Crack growth in a plate with rivet holes}

The purpose of this example is to evaluate the potential of IGABEM for crack growth. The problem is chosen from the XFEM work by Moës et al [6]. The geometry and loading conditions are illustrated in Figure $14(\theta=\pi / 4$, initial crack length $a=0.1)$. The material parameters $E=1000, \nu=0.3$. Below we compare three crack paths:

(1) the crack path, obtained by IGABEM (abbreviated as 'IGABEM'),

(2) the crack path, obtained by XFEM in [6] (abbreviated as 'XFEM(M)'),

(3) the crack path, obtained by the in-house XFEM code (abbreviated as ' $\mathrm{XFEM}^{*}$ ').

For IGABEM crack growth 12 elements are used for each circle and 3 elements for each edge 


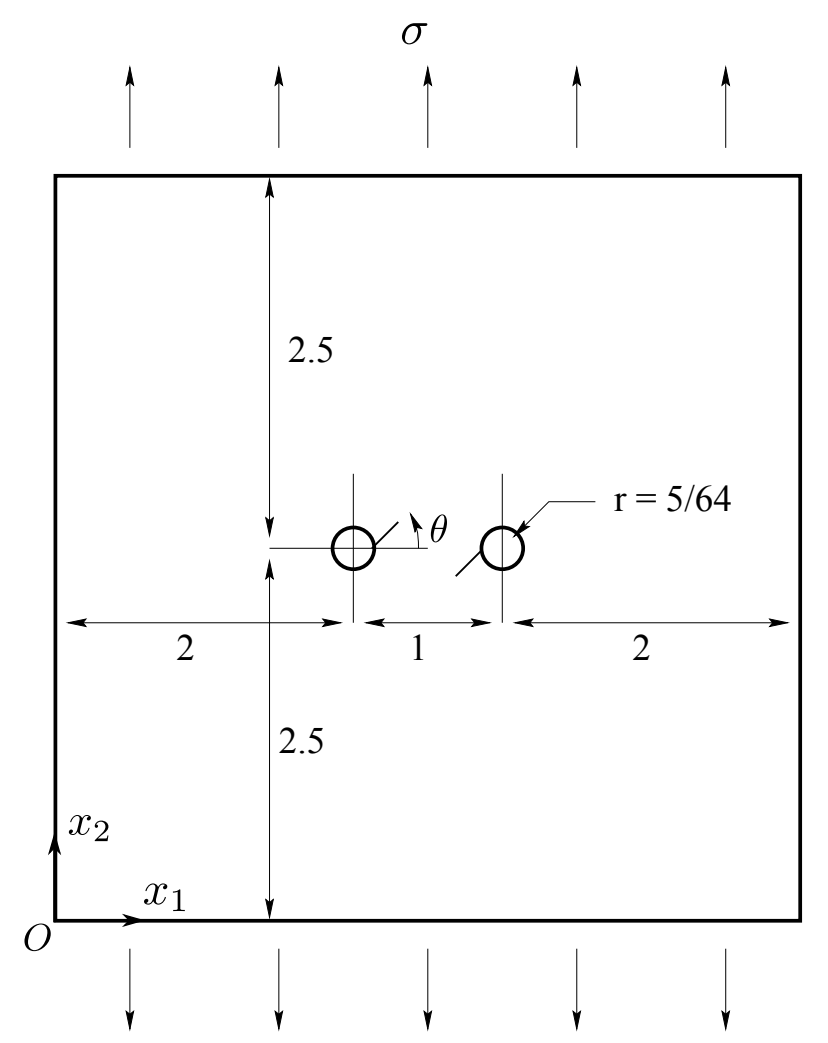

Figure 14: Physical model of rivet holes plate with initial cracks emanating from the holes. The initial crack lengths are 0.1, (Moës et al, 1999).

and for the initial cracks. The crack tip elements are further refined in the way described in section 5. We assume that each crack advances $\Delta a=0.05$ at each step, which is identical to the increment chosen in [6] for the finest mesh. We grow the crack for 16 steps.

Next, all three crack paths - (1), (2) and (3) - are compared in Figure 15. The tip positions and SIFs for the left crack in each step are further compared in Table 6. It can be observed that the tip positions and the crack paths in all three cases are quasi-identical during propagation. From Figure 16 (a) we note that SIFs display significant difference in steps $9 \sim 12$. However, the crack growth direction is defined by the ratio $K_{I I} / K_{I}$ which is shown in 16 (b) and after these values of $K_{I I} / K_{I}$ are employed into the crack growth criteria, the final difference in the crack tip positions between all three paths does not exceed the difference in the third digital sign.

\subsection{Three holes plate bending problem}

A three point bending beam with three holes is simulated to further verify the robustness and accuracy of IGABEM for crack propagation. The geometry and loading conditions are 


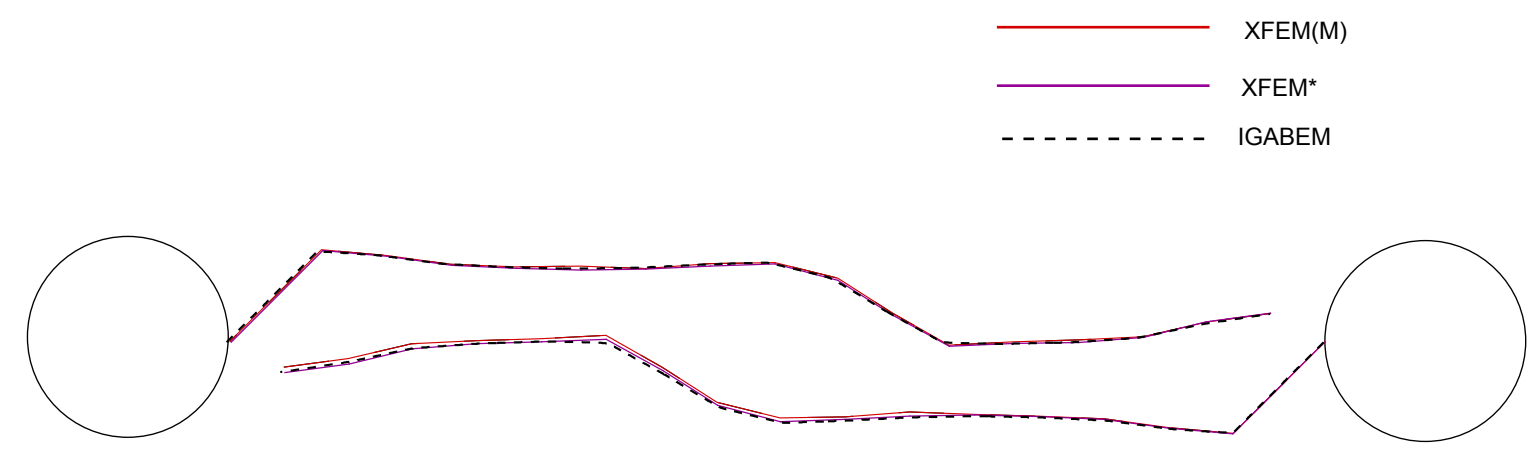

Figure 15: Crack path comparison. XFEM(M) is from Moës et al, 1999; XFEM* is from the in-house XFEM code

\begin{tabular}{|c|c|c|c|c|c|c|}
\hline & \multicolumn{2}{|c|}{ IGABEM } & \multicolumn{2}{c|}{ XFEM $^{*}$} & \multicolumn{2}{c|}{ XFEM(M) } \\
\hline Step & $x_{c}$ & $y_{c}$ & $x_{c}$ & $y_{c}$ & $x_{c}$ & $y_{c}$ \\
\hline Initial & 2.1488 & 2.5707 & 2.1488 & 2.5707 & 2.1488 & 2.5707 \\
\hline 1 & 2.1986 & 2.5665 & 2.1986 & 2.5662 & 2.1986 & 2.5663 \\
\hline 2 & 2.2481 & 2.5596 & 2.2481 & 2.5593 & 2.2481 & 2.5595 \\
\hline 3 & 2.2981 & 2.5575 & 2.2981 & 2.5570 & 2.2981 & 2.5575 \\
\hline 4 & 2.3481 & 2.5564 & 2.3480 & 2.5556 & 2.3481 & 2.5581 \\
\hline 5 & 2.3981 & 2.5573 & 2.3980 & 2.5564 & 2.3981 & 2.5562 \\
\hline 6 & 2.4480 & 2.5598 & 2.4480 & 2.5587 & 2.4480 & 2.5600 \\
\hline 7 & 2.4980 & 2.5614 & 2.4979 & 2.5604 & 2.4980 & 2.5608 \\
\hline 8 & 2.5463 & 2.5485 & 2.5463 & 2.5477 & 2.5465 & 2.5488 \\
\hline 9 & 2.5885 & 2.5217 & 2.5885 & 2.5209 & 2.5886 & 2.5219 \\
\hline 10 & 2.6324 & 2.4978 & 2.6324 & 2.4968 & 2.6321 & 2.4972 \\
\hline 11 & 2.6824 & 2.4986 & 2.6823 & 2.4990 & 2.6820 & 2.4998 \\
\hline 12 & 2.7324 & 2.5000 & 2.7323 & 2.4997 & 2.7320 & 2.5013 \\
\hline 13 & 2.7823 & 2.5035 & 2.7821 & 2.5036 & 2.7819 & 2.5037 \\
\hline 14 & 2.8311 & 2.5144 & 2.8307 & 2.5157 & 2.8306 & 2.5151 \\
\hline 15 & 2.8805 & 2.5217 & 2.8802 & 2.5223 & 2.8802 & 2.5217 \\
\hline
\end{tabular}

Table 6: Tip position for left crack tip with $\Delta a=0.05$. XFEM(M) is from Moës et al, 1999, $\mathrm{XFEM}^{*}$ is from the in-house XFEM code. The final difference in the crack tip positions between all three paths does not exceed the difference in the third digital sign 


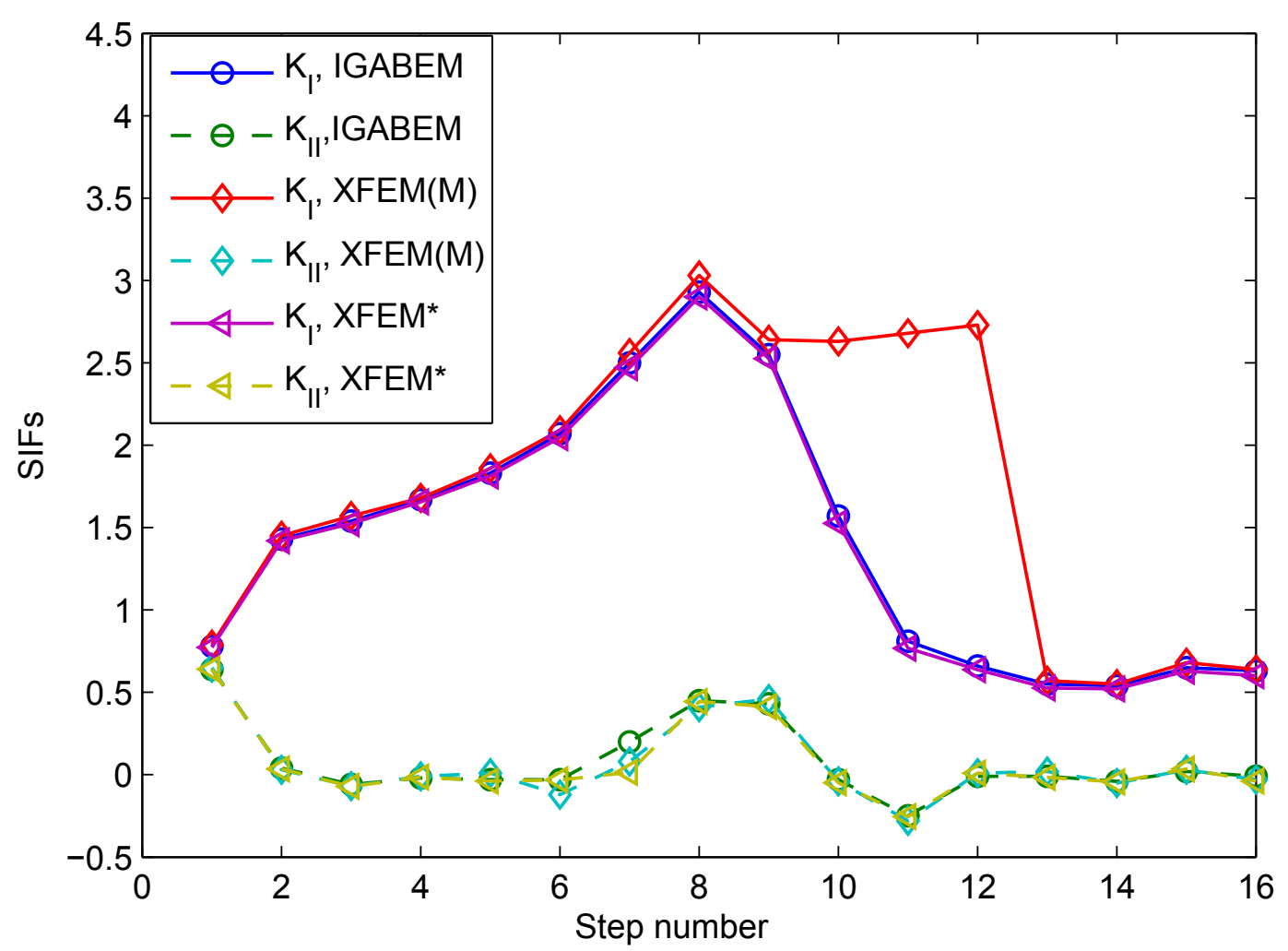

(a)

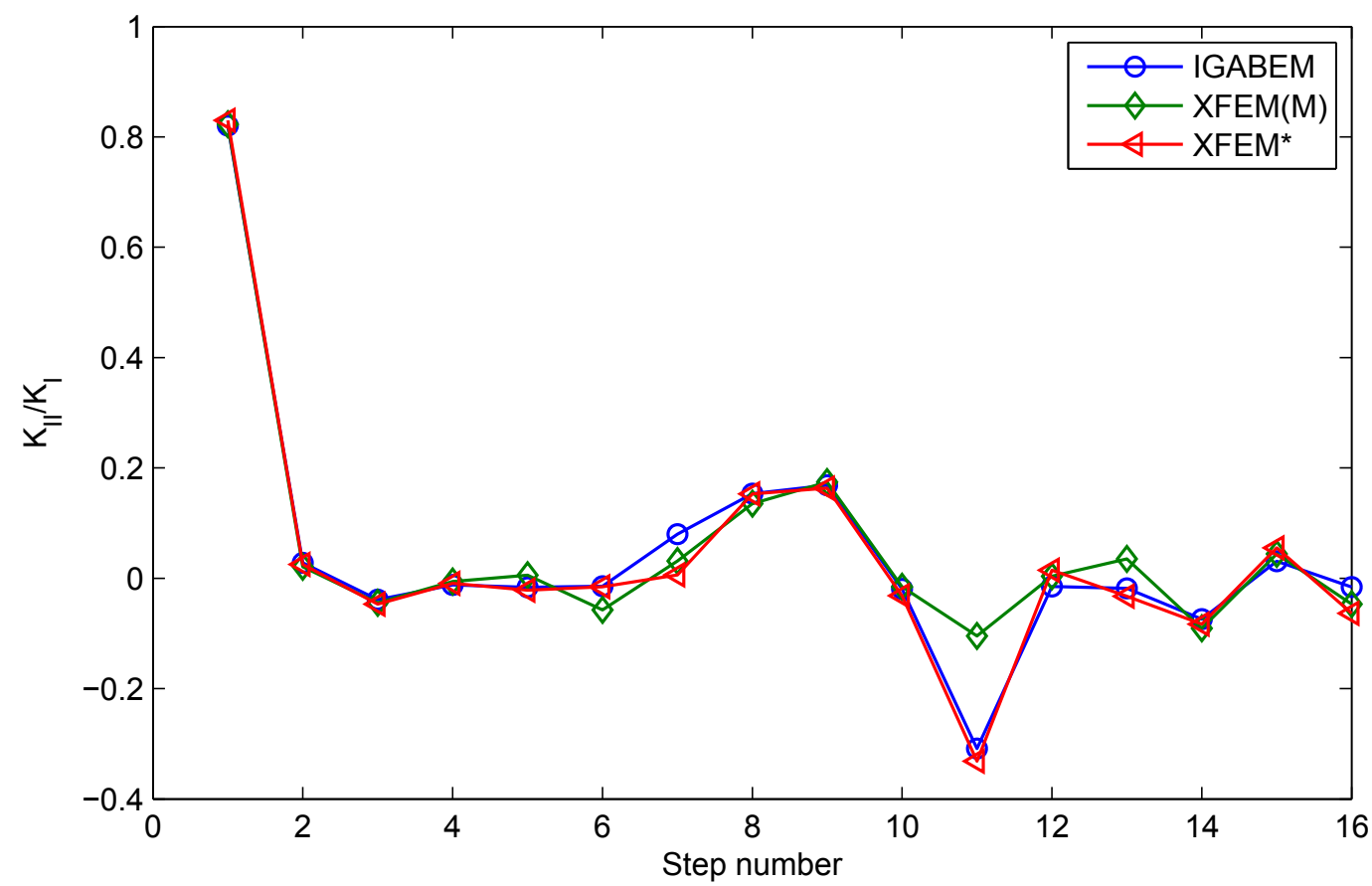

(b)

Figure 16: SIF comparison of the left crack tip for the whole process of crack propagation. $\operatorname{XFEM}(\mathrm{M})$ is from Moës et al, 1999, XFEM* is from the in-house XFEM code 


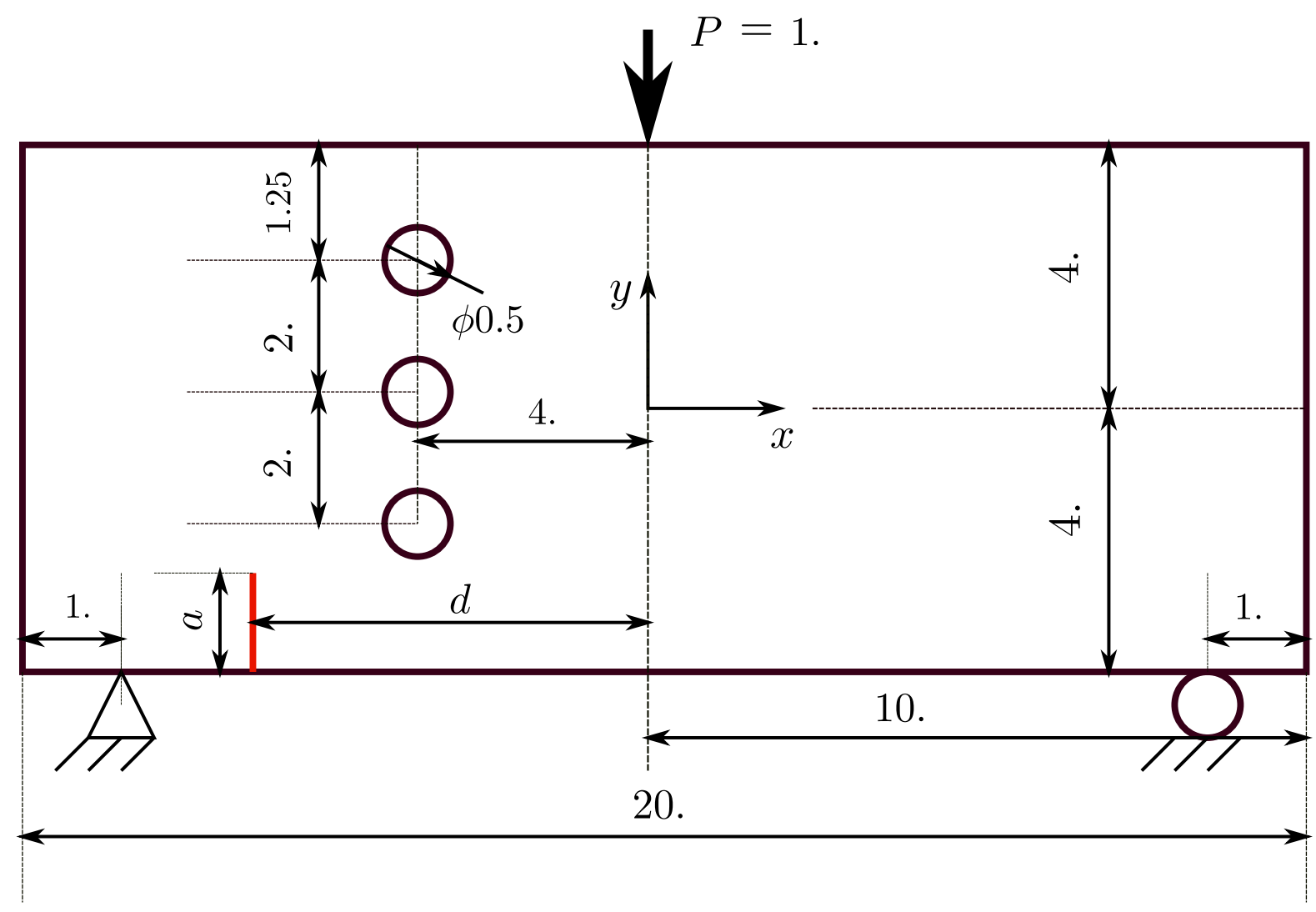

Figure 17: Physical model of the three point bending beam with 3 holes

illustrated in Figure 17. The material parameters are $E=1000, \nu=0.37$. Plane strain conditions are assumed. With variation of the position of the initial crack, different crack trajectories were obtained experimentally in [99]. Here the position of the initial crack is set as $d=5, a=1.5$. This example has been solved using XFEM and XEFG [100] as well. The crack advance $\Delta a$ is set to be 0.052 for both XFEM and IGABEM. The model is discretized by 27, 869 nodes and 55, 604 triangular elements for XFEM. And for IGABEM, 82 elements and 230 DOFs are used. Crack tip mesh refinement is used without enrichment. In [100], the XEFG model size is not given, but the crack increment $\Delta a=0.1$. Figure 18 compares the crack growth paths using all the mentioned methods. All the crack paths agree well with the experiments. Of course, due to the differences in discretization and crack increment, the numerical results do differ. It can be observed that the IGABEM reproduces slightly better the experimental crack trajectory than the XFEM for the case when the crack passes through the first hole. Figure 19 compares the SIFs from XFEM and IGABEM. We note that significant difference in SIF values and the ratio of $K_{I I} / K_{I}$ occur when the crack passes near the first hole. A possible explanation for this could be that in XFEM, the domain used for SIF extraction is allowed to be intersected 


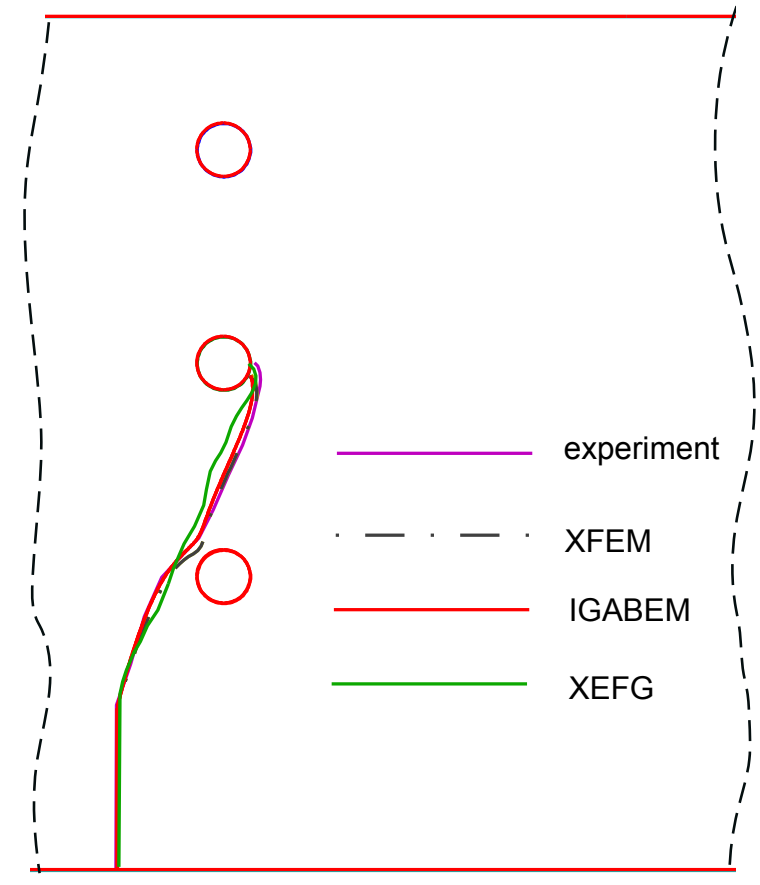

Figure 18: Crack paths (XEFG result $\Delta a=0.1$ is from Ventura et al, 2002)

with the boundary of the domain.

\subsection{Crack propagation in an open spanner}

The last example consists in simulating the failure process of an open spanner due to crack propagation, in which the geometry is taken directly from CAD. The physical configuration is shown in Figure 20. As in industrial damage tolerance assessment [86], we assume that a small defect has initiated from the surface at the area of high stress concentration obtained from an elastostatic analysis [2]. The initial geometry including the crack is given in Figure 21. The crack will grow with $\Delta a=0.1$. Figure 22 presents the deformed geometry with the crack. This example gives a straightforward illustration of the concept of seamless integration of CAD and failure analysis, since no mesh generator is required and the crack path is obtained directly from CAD.

\section{Conclusions}

A detailed procedure to model linear elastic fracture problem using the NURBS based IGABEM is proposed in this work. The dual BIEs is introduced so that cracks can be modeled in a single 


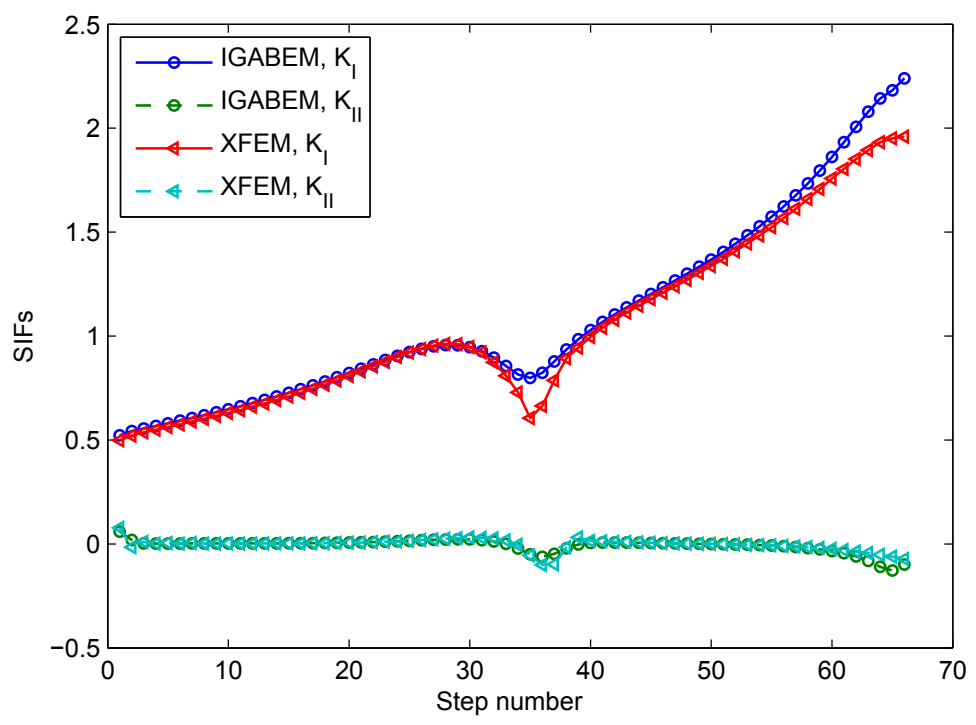

(a)

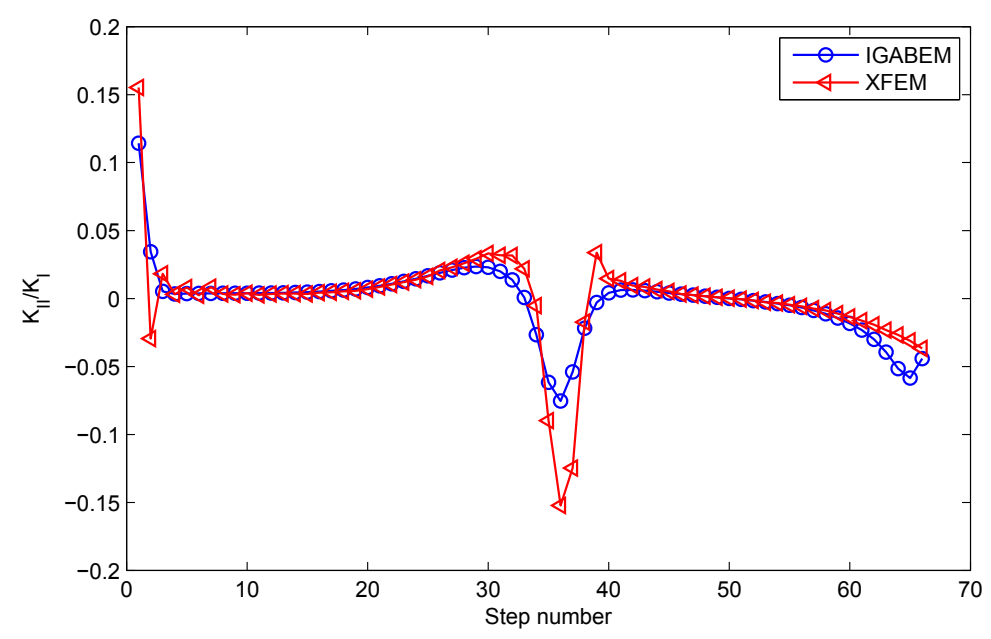

(b)

Figure 19: Comparison of the SIFs for the whole process of crack propagation

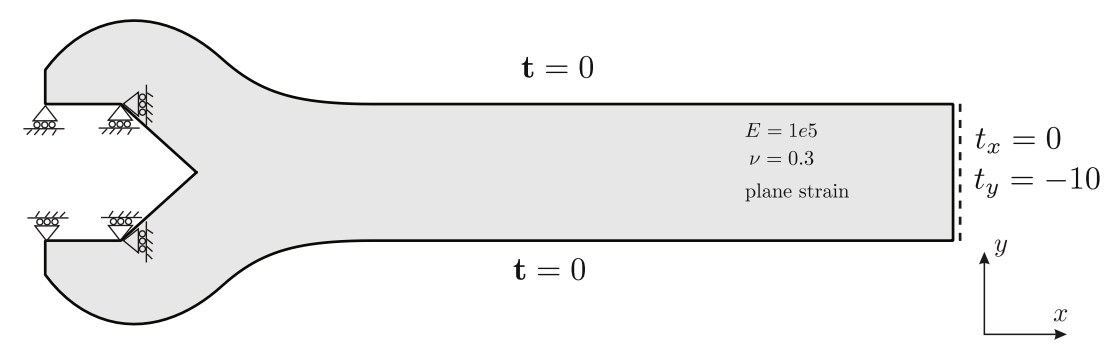

Figure 20: Boundary conditions, materials and geometry of the open spanner (Simpson et al, 2012) 


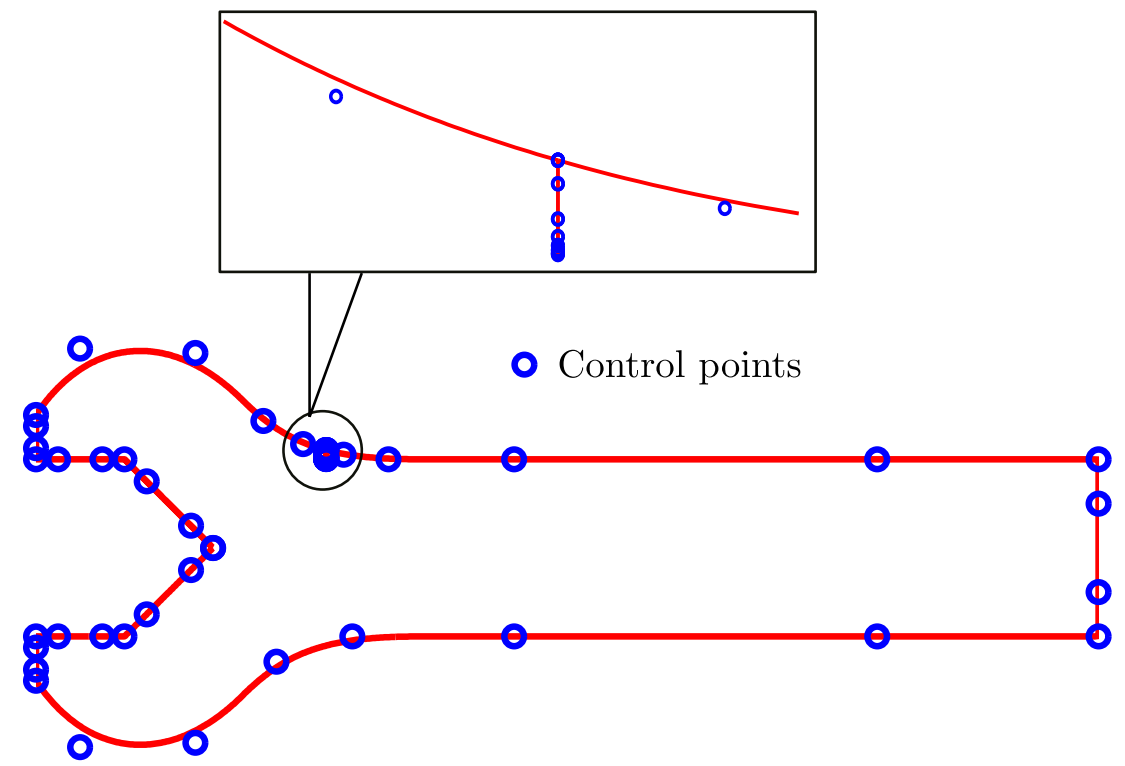

Figure 21: Control points and NURBS representation of the open spanner

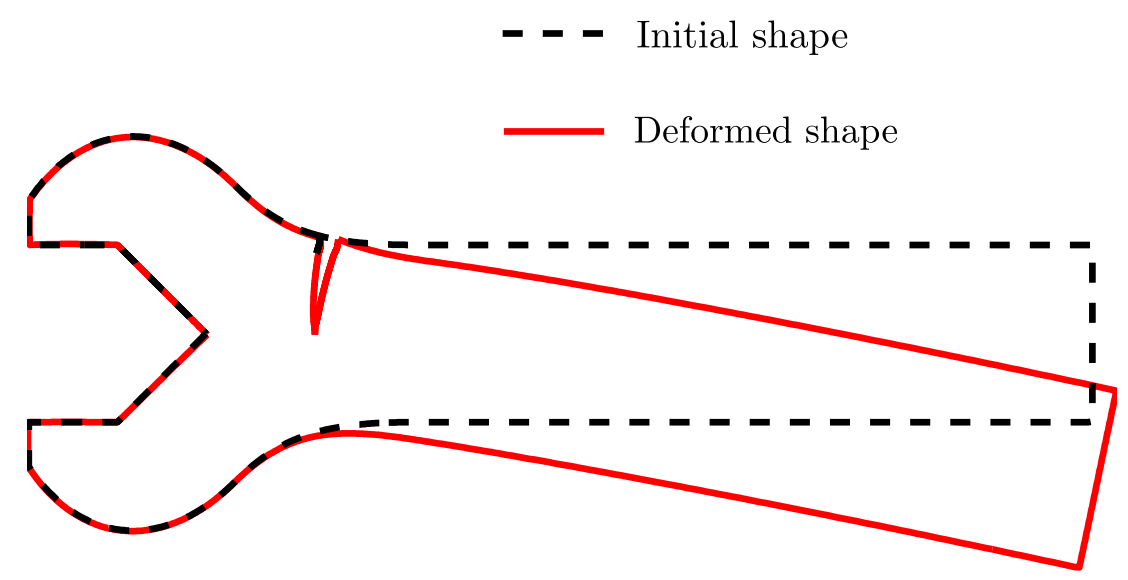

Figure 22: The deformed geometry after 10 steps of crack propagation 
domain. Different treatments for crack tip singularity are investigated including crack tip graded mesh refinement and partition of unity enrichment. The popular approaches to extract SIFs are compared in the framework of IGABEM and it proves that the $M$ integral is more efficient for SIF extraction in IGABEM. The cracks are modeled directly by NURBS, and an algorithm for modifying the NURBS curve is implemented to describe the crack propagation. Numerical examples shows that:

(1) The IGABEM can obtain a higher accuracy than Lagrange basis based BEM for the same model size or DOFs. The convergence rate in SIFs has been improved by $5 \sim 8$ times than BEM with discontinuous Lagrange basis without any treatment to the crack tip;

(2) Both crack tip graded mesh refinement and enrichment can improve the displacement field near the crack tip, and the graded mesh refinement is selected to apply in the crack growth;

(3) The proposed crack growth procedure can lead to $C^{1}$ smooth crack trajectory and agrees well with those results from XFEM.

(4) A procedure for damage tolerance assessment directly from CAD is presented, which does not require any mesh (re)generation.

The authors believe that the crack propagation in three dimensional domain would benefit more thanks to the smooth crack representation and higher order continuous NURBS basis, which would provide a distinct solution scheme for fracture analysis when compared to the idea in the framework of FEM/XFEM.

\section{Acknowledgements}

The first and last authors would like to acknowledge the financial support of the Framework Programme 7 Initial Training Network Funding under grant number 289361 'Integrating Numerical Simulation and Geometric Design Technology'. S. P. A. Bordas also thanks partial funding for his time provided by the UK Engineering and Physical Science Research Council (EPSRC) under grant EP/G069352/1 Advanced discretization strategies for 'atomistic' nano CMOS simulation; the EPSRC under grant EP/G042705/1 'Increased Reliability for Industrially Relevant Automatic Crack Growth Simulation with the eXtended Finite Element Method' and the European Research Council Starting Independent Research Grant (ERC Stg grant agreement No. 279578 ) entitled 'Towards real time multiscale simulation of cutting in non-linear materials 
with applications to surgical simulation and computer guided surgery'. E. Atroshchenko was partially supported by Fondecyt grant number 11130259 entitled 'Boundary element modeling of crack propagation in micropolar materials'.

\section{Appendix A}

The fundamental solutions for traction BIE are:

$$
\begin{aligned}
K_{i j} & =\frac{1}{4 \pi(1-\nu) r}\left[(1-2 \nu)\left(\delta_{i j} r_{, k}+\delta_{j k} r_{, i}-\delta_{i k} r_{, j}\right)+2 r_{, i} r_{, j} r_{, k}\right] n_{k}(\mathbf{s}) \\
S_{i j} & =\frac{\mu}{2 \pi(1-\nu) r^{2}}\left\{2 \frac{\partial r}{\partial n}\left[(1-2 \nu) \delta_{i k} r_{, j}+\nu\left(\delta_{i j} r_{, k}+\delta_{j k} r_{, i}\right)-4 r_{, i} r_{, j} r_{, k}\right]\right. \\
& +2 \nu\left(n_{i} r_{, j} r_{, k}+n_{k} r_{, i} r_{, j}\right)-(1-4 \nu) \delta_{i k} n_{j} \\
& \left.+(1-2 \nu)\left(2 n_{j} r_{, i} r_{, k}+\delta_{i j} n_{k}+\delta_{j k} n_{i}\right)\right\} n_{k}(\mathbf{s})
\end{aligned}
$$

Now we present the SST formula for the hyper-singular integral as follows. Expanding the components of distance between field and source points as Taylor series in parent space gives:

$$
\begin{aligned}
x_{i}-s_{i} & =\left.\frac{\mathrm{d} x_{i}}{\mathrm{~d} \hat{\xi}}\right|_{\hat{\xi}=\hat{\xi}_{s}}\left(\hat{\xi}-\hat{\xi_{s}}\right)+\left.\frac{\mathrm{d}^{2} x_{i}}{\mathrm{~d} \hat{\xi}^{2}}\right|_{\hat{\xi}=\hat{\xi}_{s}} \frac{\left(\hat{\xi}-\hat{\xi_{s}}\right)^{2}}{2}+\cdots \\
& :=A_{i}\left(\hat{\xi}-\hat{\xi}_{s}\right)+B_{i}\left(\hat{\xi}-\hat{\xi}_{s}\right)^{2}+\cdots \\
& =A_{i} \delta+B_{i} \delta^{2}+O\left(\delta^{3}\right)
\end{aligned}
$$

and

$$
\begin{aligned}
A & :=\left(\sum_{k=1}^{2} A_{k}^{2}\right)^{\frac{1}{2}} \\
C & :=\sum_{k=1}^{2} A_{k} B_{k}
\end{aligned}
$$


The first and second derivatives are:

$$
\begin{aligned}
\frac{\mathrm{d} x_{i}}{\mathrm{~d} \xi} & =\frac{\mathrm{d} N_{a}}{\mathrm{~d} \xi} x_{i}^{a} \\
\frac{\mathrm{d}^{2} x_{i}}{\mathrm{~d} \xi^{2}} & =\frac{\mathrm{d}^{2} N_{a}}{\mathrm{~d} \xi^{2}} x_{i}^{a} \\
\frac{\mathrm{d} x_{i}}{\mathrm{~d} \hat{\xi}} & =\frac{\mathrm{d} x_{i}}{\mathrm{~d} \xi} \frac{\mathrm{d} \xi}{\mathrm{d} \hat{\xi}} \\
\frac{\mathrm{d}^{2} x_{i}}{\mathrm{~d} \hat{\xi}^{2}} & =\frac{\mathrm{d}^{2} x_{i}}{\mathrm{~d} \xi^{2}}\left(\frac{\mathrm{d} \xi}{\mathrm{d} \hat{\xi}}\right)^{2}
\end{aligned}
$$

The derivative $r_{, i}$ can be expressed as

$$
\begin{aligned}
r_{, i} & =\frac{x_{i}-s_{i}}{r}=\frac{A_{i}}{A}+\left(B_{i} A-A_{i} \frac{A_{k} B_{k}}{A^{3}}\right) \delta+O\left(\delta^{2}\right) \\
& :=d_{i 0}+d_{i 1} \delta+O\left(\delta^{2}\right)
\end{aligned}
$$

The term $1 / r^{2}$ can be expressed as

$$
\begin{aligned}
\frac{1}{r^{2}} & =\frac{1}{A^{2} \delta^{2}}-\frac{2 C}{A^{4} \delta}+O(1) \\
& :=\frac{S_{-2}}{\delta^{2}}+\frac{S_{-1}}{\delta}+O(1)
\end{aligned}
$$

The component of Jacobian from parametric space to physical space can be expressed as:

$$
\begin{aligned}
J_{1}(\xi) & =J_{10}\left(\xi_{s}\right)+J_{11}\left(\xi_{s}\right)\left(\xi-\xi_{s}\right)+O\left(\left(\xi-\xi_{s}\right)^{2}\right) \\
& =J_{10}\left(\xi_{s}\right)+\left.\frac{\mathrm{d} \xi}{\mathrm{d} \hat{\xi}}\right|_{\xi=\xi_{s}} J_{11}\left(\xi_{s}\right) \delta+O\left(\delta^{2}\right) \\
J_{2}(\xi) & =J_{20}\left(\xi_{s}\right)+J_{21}\left(\xi_{s}\right)\left(\xi-\xi_{s}\right)+O\left(\left(\xi-\xi_{s}\right)^{2}\right) \\
& =J_{10}\left(\xi_{s}\right)+\left.\frac{\mathrm{d} \xi}{\mathrm{d} \hat{\xi}}\right|_{\xi=\xi_{s}} J_{21}\left(\xi_{s}\right) \delta+O\left(\delta^{2}\right)
\end{aligned}
$$

i.e.,

$$
J_{k}(\xi):=J_{k 0}\left(\xi_{s}\right)+\left.\frac{\mathrm{d} \xi}{\mathrm{d} \hat{\xi}}\right|_{\xi=\xi_{s}} J_{k 1}\left(\xi_{s}\right) \delta+O\left(\delta^{2}\right)
$$


and we note that

$$
\begin{aligned}
J(\xi) & =\sqrt{J_{1}^{2}(\xi)+J_{2}^{2}(\xi)}=\sqrt{\left(\frac{\mathrm{d} y}{\mathrm{~d} \xi}\right)^{2}+\left(-\frac{\mathrm{d} x}{\mathrm{~d} \xi}\right)^{2}} \\
\mathbf{n}(\xi) & =\left[\frac{\mathrm{d} y}{\mathrm{~d} \xi},-\frac{\mathrm{d} x}{\mathrm{~d} \xi}\right] \\
\text { i.e., } & \\
n_{k}(\xi) & =J_{k}(\xi) / J(\xi)
\end{aligned}
$$

And the NURBS basis function is also expanded as:

$$
\begin{aligned}
N_{a}(\hat{\xi}) & =N_{a}\left(\hat{\xi}_{s}\right)+\left.\frac{\mathrm{d} N_{a}}{\mathrm{~d} \xi}\right|_{\xi=\xi_{s}}\left(\xi-\xi_{s}\right)+\cdots \\
& =N_{a}\left(\hat{\xi}_{s}\right)+\left.\left.\frac{\mathrm{d} N_{a}}{\mathrm{~d} \xi}\right|_{\xi=\xi_{s}} \frac{\mathrm{d} \xi}{\mathrm{d} \hat{\xi}}\right|_{\hat{\xi}=\hat{\xi}_{s}} \delta+\cdots \\
& :=N_{a 0}\left(\hat{\xi}_{s}\right)+\left.N_{a 1}\left(\hat{\xi}_{s}\right) \frac{\mathrm{d} \xi}{\mathrm{d} \hat{\xi}}\right|_{\hat{\xi}=\hat{\xi}_{s}} \delta+O\left(\delta^{2}\right)
\end{aligned}
$$

The detail form of hyper-singular kernel $S_{i j}$ is (plane strain)

$$
\begin{aligned}
S_{i j}(\mathbf{s}, \mathbf{x}) & =\frac{\mu}{2 \pi(1-\nu) r^{2}}\left\{2 \frac{\partial r}{\partial n}\left[(1-\nu) \delta_{i k} r_{, j}+\nu\left(\delta_{i j} r_{, k}+\delta_{j k} r_{, i}-4 r_{, i} r_{, j} r_{, k}\right)\right]\right. \\
& +2 \nu\left(n_{i} r_{, j} r_{, k}+n_{k} r_{, i} r_{, j}\right)-(1-4 \nu) \delta_{i k} n_{j} \\
& \left.+(1-2 \nu)\left(2 n_{j} r_{, i} r_{, k}+\delta_{i j} n_{k}+\delta_{j k} n_{i}\right)\right\} n_{k}\left(\hat{\xi}_{s}\right) \\
& :=\frac{1}{r^{2}} h(\hat{\xi})
\end{aligned}
$$

Noting that $n_{k}(\xi)=J_{k}(\xi) / J(\xi)$, Use the above expansions to rewrite $h(\xi)$ as:

$$
\begin{gathered}
h(\hat{\xi})=\frac{h_{0}\left(\hat{\xi_{s}}\right)}{J(\xi)}+\frac{h_{1}\left(\hat{\xi}_{s}\right)}{J(\xi)} \delta+O\left(\delta^{2}\right) \\
h_{0}\left(\hat{\xi}_{s}\right)=\left(2 \nu\left(J_{i 0} d_{j 0} d_{k 0}+J_{k 0} d_{i 0} d_{j 0}\right)+(1-2 \nu)\left(2 J_{j 0} d_{i 0} d_{k 0}+\delta_{i j} J_{k 0}+\delta_{j k} J_{i 0}\right)\right. \\
\left.+(1-4 \nu) \delta_{i k} J_{j 0}\right) \frac{\mu}{2 \pi(1-\nu)} n_{k}\left(\hat{\xi}_{s}\right)
\end{gathered}
$$




$$
\begin{aligned}
h_{1}\left(\hat{\xi}_{s}\right) & =\left[2\left(d_{l 1} J_{l 0}+d_{l 0} J_{l 1}\right)\left((1-2 \nu) \delta_{i k} d_{j 0}+\nu\left(\delta_{i j} d_{k 0}+\delta_{j k} d_{i 0}\right)-4 d_{i 0} d_{j 0} d_{k 0}\right)\right. \\
& +2 \nu\left(J_{i 0}\left(d_{j 1} d_{k 0}+d_{j 0} d_{k 1}\right)+J_{i 1} d_{j 0} d_{k 0}+J_{k 0}\left(d_{i 1} d_{j 0}+d_{i 0} d_{j 1}\right)+J_{k 1} d_{i 0} d_{j 0}\right) \\
& +(1-2 \nu)\left(2\left(J_{j 1} d_{i 0} d_{k 0}+J_{j 0}\left(d_{i 1} d_{k 0}+d_{i 0} d_{k 1}\right)\right)+\delta_{i j} J_{k 1}+\delta_{j k} J_{i 1}\right) \\
& \left.-(1-4 \nu) \delta_{i k} J_{j 1}\right] \frac{\mu}{2 \pi(1-\nu)} n_{k}\left(\hat{\xi}_{s}\right)
\end{aligned}
$$

Thus,

$$
\begin{aligned}
h(\hat{\xi}) N_{a}(\hat{\xi}) J(\hat{\xi}) & =\left(h_{0}\left(\hat{\xi}_{s}\right)+h_{1}\left(\hat{\xi}_{s}\right) \delta+O\left(\delta^{2}\right)\right)\left(N_{a 0}\left(\hat{\xi}_{s}\right)+\left.\frac{\mathrm{d} \xi}{\mathrm{d} \hat{\xi}}\right|_{\hat{\xi}=\hat{\xi}_{s}} N_{a 1}\left(\hat{\xi}_{s}\right) \delta+O\left(\delta^{2}\right)\right) \\
& =h_{0} N_{a 0}+\left(h_{1} N_{a 0}+\left.h_{0} N_{a 1} \frac{\mathrm{d} \xi}{\mathrm{d} \hat{\xi}}\right|_{\hat{\xi}=\hat{\xi}_{s}}\right) \delta+O\left(\delta^{2}\right) \\
F\left(\hat{\xi}_{s}, \hat{\xi}\right)= & \frac{1}{r^{2}\left(\hat{\xi}_{s}, \hat{\xi}\right)} h(\hat{\xi}) N_{a}(\hat{\xi}) J(\hat{\xi}) \\
= & \left(\frac{S_{-2}}{\delta^{2}}+\frac{S_{-1}}{\delta}+O(1)\right)\left(h_{0} N_{a 0}+\left(h_{1} N_{a 0}+\left.h_{0} N_{a 1} \frac{\mathrm{d} \xi}{\mathrm{d} \hat{\xi}}\right|_{\hat{\xi}=\hat{\xi}_{s}}\right) \delta+O\left(\delta^{2}\right)\right) \\
= & \frac{S_{-2} h_{0} N_{a 0}}{\delta^{2}}+\frac{S_{-1} h_{0} N_{a 0}+S_{-2}\left(h_{1} N_{a 0}+\left.h_{0} N_{a 1} \frac{\mathrm{d} \xi}{\mathrm{d} \hat{\xi}}\right|_{\hat{\xi}=\hat{\xi}_{s}}\right)}{\delta}+O(1) \\
& :=\frac{F_{-2}}{\delta^{2}}+\frac{F_{-1}}{\delta}+O(1)
\end{aligned}
$$

\section{Appendix B}

Once the $J_{1}$ and $J_{2}$ are evaluated properly, $K_{I}$ and $K_{I I}$ can be found easily. Since

$$
\begin{aligned}
& J_{1}=\frac{K_{I}^{2}+K_{I I}^{2}}{E^{\prime}} \\
& J_{2}=-\frac{2 K_{I} K_{I I}}{E^{\prime}}
\end{aligned}
$$

where $E^{\prime}=E /\left(1-\nu^{2}\right)$ for plane strain condition. And $K_{I}$ and $K_{I I}$ can be solved as [92]:

$$
\begin{aligned}
& K_{I}= \pm\left\{\frac{E^{\prime} J_{1}}{2}\left[1 \pm\left(1-\left(\frac{J_{2}}{J_{1}}\right)^{2}\right)^{1 / 2}\right]\right\}^{1 / 2} \\
& K_{I I}= \pm\left\{\frac{E^{\prime} J_{1}}{2}\left[1 \mp\left(1-\left(\frac{J_{2}}{J_{1}}\right)^{2}\right)^{1 / 2}\right]\right\}^{1 / 2}
\end{aligned}
$$


The signs of $K_{I}$ and $K_{I I}$ correspond to the signs of crack opening displacement $\llbracket u_{1} \rrbracket$ and $\llbracket u_{2} \rrbracket$, respectively. If $\llbracket u_{1} \rrbracket>0, K_{I}>0$. The term in brace can be determined as :

$$
\begin{aligned}
& \text { if }\left|\llbracket u_{1} \rrbracket\right| \geq\left|\llbracket u_{2} \rrbracket\right|, \text { take+ } \\
& \text { if }\left|\llbracket u_{1} \rrbracket\right|<\left|\llbracket u_{2} \rrbracket\right|, \text { take- }
\end{aligned}
$$

Combined with Equation 65a, the following relationship can be obtained for the $M$ integral,

$$
M^{(1,2)}=\frac{2}{E^{\prime}}\left(K_{I}^{(1)} K_{I}^{(2)}+K_{I I}^{(1)} K_{I I}^{(2)}\right)
$$

Let state 2 be the pure mode $I$ asymptotic fields with $K_{I}^{(2)}=1, K_{I I}^{(2)}=0$ and $K_{I}$ in real state 1 can be found as

$$
K_{I}^{(1)}=\frac{2}{E^{\prime}} M^{(1, \text { mode } I)}
$$

The $K_{I I}$ can be given in a similar fashion.

The auxiliary stress field $\sigma_{i j}^{(2)}$ and displacement field $u_{j}^{(2)}$ are given as:

$$
\begin{aligned}
\sigma_{x x}(r, \theta)= & \frac{K_{I}^{(2)}}{\sqrt{2 \pi r}} \cos \frac{\theta}{2}\left(1-\sin \frac{\theta}{2} \sin \frac{3 \theta}{2}\right)-\frac{K_{I I}^{(2)}}{\sqrt{2 \pi r}} \sin \frac{\theta}{2}\left(2+\cos \frac{\theta}{2} \cos \frac{3 \theta}{2}\right) \\
\sigma_{y y}(r, \theta)= & \frac{K_{I}^{(2)}}{\sqrt{2 \pi r}} \cos \frac{\theta}{2}\left(1+\sin \frac{\theta}{2} \sin \frac{3 \theta}{2}\right)+\frac{K_{I I}^{(2)}}{\sqrt{2 \pi r}} \sin \frac{\theta}{2} \cos \frac{\theta}{2} \cos \frac{3 \theta}{2} \\
\tau_{x y}(r, \theta)= & \frac{K_{I}^{(2)}}{\sqrt{2 \pi r}} \sin \frac{\theta}{2} \cos \frac{\theta}{2} \cos \frac{3 \theta}{2}+\frac{K_{I I}^{(2)}}{\sqrt{2 \pi r}} \cos \frac{\theta}{2}\left(1-\sin \frac{\theta}{2} \sin \frac{3 \theta}{2}\right) \\
u_{x}(r, \theta)= & \frac{K_{I}}{2 \mu} \sqrt{\frac{r}{2 \pi}} \cos \frac{\theta}{2}\left(\kappa-1+2 \sin ^{2} \frac{\theta}{2}\right) \\
+ & \frac{(1+\nu) K_{I I}}{E} \sqrt{\frac{r}{2 \pi}} \sin \frac{\theta}{2}\left(\kappa+1+2 \cos ^{2} \frac{\theta}{2}\right) \\
u_{y}(r, \theta)= & \frac{K_{I}}{2 \mu} \sqrt{\frac{r}{2 \pi}} \sin \frac{\theta}{2}\left(\kappa+1-2 \cos ^{2} \frac{\theta}{2}\right) \\
& +\frac{(1+\nu) K_{I I}}{E} \sqrt{\frac{r}{2 \pi}} \cos \frac{\theta}{2}\left(1-\kappa+2 \sin ^{2} \frac{\theta}{2}\right)
\end{aligned}
$$

where $(r, \theta)$ are the crack tip polar coordinates and

$$
\mu=\frac{E}{2(1+\nu)}
$$




$$
\kappa= \begin{cases}3-4 \nu, & \text { Plane strain } \\ (1-\nu) /(3+\nu), & \text { Plane stress }\end{cases}
$$

The auxiliary strain field can be obtained by differentiating $u_{j}$ with respect to the physical coordinate.

\section{References}

[1] T J R Hughes, J A Cottrell, and Y Bazilevs. Isogeometric analysis: CAD, finite elements, NURBS, exact geometry and mesh refinement. Computer Methods in Applied Mechanics and Engineering, 194(39-41):4135-4195, 2005.

[2] R N Simpson, S P A Bordas, J Trevelyan, and T Rabczuk. A two-dimensional Isogeometric Boundary Element Method for elastostatic analysis. Computer Methods in Applied Mechanics and Engineering, 209-212(0):87-100, 2012.

[3] M A Scott, R N Simpson, J A Evans, S Lipton, S P A Bordas, T J R Hughes, and T W Sederberg. Isogeometric boundary element analysis using unstructured T-splines. Computer Methods in Applied Mechanics and Engineering, 254(0):197-221, 2013.

[4] P.O. Bouchard, F. Bay, Y. Chastel, and I. Tovena. Crack propagation modelling using an advanced remeshing technique. Computer Methods in Applied Mechanics and Engineering, 189(3):723-742, sep 2000.

[5] S P A Bordas, T Rabczuk, and G Zi. Three-dimensional crack initiation, propagation, branching and junction in non-linear materials by an extended meshfree method without asymptotic enrichment. Engineering Fracture Mechanics, 75(5):943-960, 2008.

[6] N Moës, J Dolbow, and T Belytschko. A finite element method for crack growth without remeshing. International Journal for Numerical Methods in Engineering, 46(1):131-150, 1999.

[7] A P Cisilino and M H Aliabadi. Dual boundary element assessment of three-dimensional fatigue crack growth. Engineering Analysis with Boundary Elements, 28(9):1157-1173, 2004 . 
[8] E De Luycker, D J Benson, T Belytschko, Y Bazilevs, and M C Hsu. X-FEM in isogeometric analysis for linear fracture mechanics. International Journal for Numerical Methods in Engineering, 87(6):541-565, 2011.

[9] S Ghorashi, N Valizadeh, and S Mohammadi. Extended isogeometric analysis for simulation of stationary and propagating cracks. International Journal for Numerical Methods in Engineering, 89(9):1069-1101, 2012.

[10] V P Nguyen, P Kerfriden, and S P A Bordas. Two- and three-dimensional isogeometric cohesive elements for composite delamination analysis. Composites Part B: Engineering, 60:193-212, apr 2014.

[11] M Duflot and S P A Bordas. A posteriori error estimation for extended finite elements by an extended global recovery. International Journal for Numerical Methods in Engineering, 76(8):1123-1138, 2008.

[12] S P A Bordas and M Duflot. Derivative recovery and a posteriori error estimate for extended finite elements. Computer Methods in Applied Mechanics and Engineering, 196(3536):3381-3399, 2007.

[13] O A González-Estrada, E Nadal, J J Ródenas, P Kerfriden, S P A Bordas, and F J Fuenmayor. Mesh adaptivity driven by goal-oriented locally equilibrated superconvergent patch recovery. Computational Mechanics, 53(5):957-976, 2014.

[14] J J Ródenas, O A González-Estrada, J E Tarancón, and F J Fuenmayor. A recovery-type error estimator for the extended finite element method based on singular + smooth stress field splitting. International Journal for Numerical Methods in Engineering, 76(4):545$571,2008$.

[15] O Goury. Computational time savings in multiscale fracture mechanics using model order reduction. PhD thesis, Cardiff University, 2015.

[16] A Akbari Rahimabadi. Error Controlled Adaptive Multiscale Method for Fracture in Polycrystalline Materials. PhD thesis, Cardiff University, 2014.

[17] T Rabczuk, S Bordas, and G Zi. On three-dimensional modelling of crack growth using partition of unity methods. Computers \&3 Structures, 88(23-24):1391-1411, dec 2010. 
[18] A Becker. The Boundary Element Methods in Engineering. McGraw-Hill Book Company, 1992.

[19] G E Blandford, A R Ingraffea, and J A Liggett. Two-dimensional stress intensity factor computations using the boundary element method. International Journal for Numerical Methods in Engineering, 17(3):387-404, 1981.

[20] M D Snyder and T A Cruse. Boundary-integral equation analysis of cracked anisotropic plates. International Journal of Fracture, 11(2):315-328, 1975.

[21] H Hong and J Chen. Derivations of Integral Equations of Elasticity. Journal of Engineering Mechanics, 114(6):1028-1044, 1988.

[22] A Portela, M H Aliabadi, and D P Rooke. The dual boundary element method: Effective implementation for crack problems. International Journal for Numerical Methods in Engineering, 33(6):1269-1287, 1992.

[23] Y Mi and M H Aliabadi. Dual boundary element method for three-dimensional fracture mechanics analysis. Engineering Analysis with Boundary Elements, 10(2):161-171, 1992.

[24] A Portela. Dual boundary-element method: Simple error estimator and adaptivity. International Journal for Numerical Methods in Engineering, 86(12):1457-1480, 2011.

[25] S L Crouch. Solution of plane elasticity problems by the displacement discontinuity method. I. Infinite body solution. International Journal for Numerical Methods in Engineering, 10(2):301-343, 1976.

[26] J Dominguez and M P Ariza. A direct traction BIE approach for three-dimensional crack problems. Engineering Analysis with Boundary Elements, 24(10):727-738, 2000.

[27] P Partheymüller, M Haas, and G Kuhn. Comparison of the basic and the discontinuity formulation of the 3D-dual boundary element method. Engineering Analysis with Boundary Elements, 24(10):777-788, 2000.

[28] S Li, M E Mear, and L Xiao. Symmetric weak-form integral equation method for threedimensional fracture analysis. Computer Methods in Applied Mechanics and Engineering, 151(3-4):435-459, 1998. 
[29] A Frangi. Fracture propagation in 3D by the symmetric Galerkin boundary element method. International Journal of Fracture, 116(4):313-330, 2002.

[30] A Sutradhar and G H Paulino. Symmetric Galerkin boundary element computation of T-stress and stress intensity factors for mixed-mode cracks by the interaction integral method. Engineering Analysis with Boundary Elements, 28(11):1335-1350, 2004.

[31] M Bonnet, G Maier, and C Polizzoto. Symmetric Galerkin boundary element method. Appl. Mech. Rev., 51:669-704, 1998.

[32] G P Nikishkov, J H Park, and S N Atluri. SGBEM-FEM alternating method for analyzing 3D non-planar cracks and their growth in structural components. Computer Modeling in Engineering \&3 Sciences, 2(3):401-422, 2001.

[33] B Aour, O Rahmani, and M Nait-Abdelaziz. A coupled FEM/BEM approach and its accuracy for solving crack problems in fracture mechanics. International Journal of Solids and Structures, 44(7-8):2523-2539, 2007.

[34] L Dong and Atluri S N. Fracture \& fatigue analyses: SGBEM-FEM or XFEM? part 2: 3D solids. Computer Modeling in Engineering \&3 Sciences, 90(5):3379-3413, 2013.

[35] K M Liew, Y Cheng, and S Kitipornchai. Analyzing the 2D fracture problems via the enriched boundary element-free method. International Journal of Solids and Structures, 44(11-12):4220-4233, 2007.

[36] S Natarajan and C Song. Representation of singular fields without asymptotic enrichment in the extended finite element method. International Journal for Numerical Methods in Engineering, 96(13):813-841, 2013.

[37] R D Henshell and K G Shaw. Crack tip finite elements are unnecessary. International Journal for Numerical Methods in Engineering, 9(3):495-507, 1975.

[38] Y Mi and M H Aliabadi. Discontinuous crack-tip elements: Application to 3D boundary element method. International Journal of Fracture, 67(3):R67-R71, 1994.

[39] J Martinez and J Dominguez. On the use of quarter-point boundary elements for stress intensity factor computations. International Journal for Numerical Methods in Engineering, 20(10):1941-1950, 1984. 
[40] B L Karihaloo and Q Z Xiao. Accurate determination of the coefficients of elastic crack tip asymptotic field by a hybrid crack element with p-adaptivity. Engineering Fracture Mechanics, 68(15):1609-1630, 2001.

[41] N G Zamani and W Sun. A direct method for calculating the stress intensity factor in BEM. Engineering Analysis with Boundary Elements, 11(4):285-292, 1993.

[42] M Lan, H Waisman, and I Harari. A direct analytical method to extract mixed-mode components of strain energy release rates from Irwin's integral using extended finite element method. International Journal for Numerical Methods in Engineering, 95(12):1033-1052, 2013.

[43] N Muthu, B G Falzon, S K Maiti, and S Khoddam. Modified crack closure integral technique for extraction of $\{\mathrm{SIFs}\}$ in meshfree methods. Finite Elements in Analysis and Design, 78:25-39, 2014.

[44] A Portela, M H Aliabadi, and D P Rooke. Dual boundary element analysis of cracked plates: singularity subtraction technique. International Journal of Fracture, 55(1):17-28, 1992.

[45] R H Rigby and M H Aliabadi. Decomposition of the mixed-mode J-integral-revisited. International Journal of Solids and Structures, 35(17):2073-2099, 1998.

[46] J F Yau, S S Wang, and H T Corten. A mixed-mode crack analysis of isotropic solids using conservation laws of elasticity. Journal of Applied Mechanics, 47:333-341, 1980.

[47] J H Chang and D J Wu. Stress intensity factor computation along a non-planar curved crack in three dimensions. International Journal of Solids and Structures, 44(2):371-386, 2007.

[48] K N Shivakumar and I S Raju. An equivalent domain integral method for threedimensional mixed-mode fracture problems. Engineering Fracture Mechanics, 42(6):935$959,1992$.

[49] M Gosz and B Moran. An interaction energy integral method for computation of mixedmode stress intensity factors along non-planar crack fronts in three dimensions. Engineering Fracture Mechanics, 69(3):299-319, 2002. 
[50] I Akkerman, Y Bazilevs, V M Calo, T J R Hughes, and S Hulshoff. The role of continuity in residual-based variational multiscale modeling of turbulence. Computational Mechanics, 41(3):371-378, 2008.

[51] Y Bazilevs, V M Calo, Y Zhang, and T J R Hughes. Isogeometric Fluid-structure Interaction Analysis with Applications to Arterial Blood Flow. Computational Mechanics, $38(4-5): 310-322,2006$.

[52] F Auricchio, L B da Veiga, C Lovadina, and A Reali. The importance of the exact satisfaction of the incompressibility constraint in nonlinear elasticity: mixed FEMs versus NURBS-based approximations. Computer Methods in Applied Mechanics and Engineering, 199(5-8):314-323, 2010.

[53] D J Benson, Y Bazilevs, M C Hsu, and T J R Hughes. Isogeometric shell analysis: The Reissner-Mindlin shell. Computer Methods in Applied Mechanics and Engineering, $199(5-8): 276-289,2010$.

[54] M J Borden, C V Verhoosel, M A Scott, T J R Hughes, and C M Landis. A phase-field description of dynamic brittle fracture. Computer Methods in Applied Mechanics and Engineering, 217-220(0):77-95, 2012.

[55] Y Bazilevs, V M Calo, J A Cottrell, J A Evans, T J R Hughes, S Lipton, M A Scott, and T W Sederberg. Isogeometric analysis using T-splines. Computer Methods in Applied Mechanics and Engineering, 199(5-8):229-263, 2010.

[56] M A Scott, X Li, T W Sederberg, and T J R Hughes. Local refinement of analysis-suitable T-splines. Computer Methods in Applied Mechanics and Engineering, 213-216(0):206-222, 2012.

[57] J Deng, F Chen, X Li, C Hu, W Tong, Z Yang, and Y Feng. Polynomial splines over hierarchical T-meshes. Graphical Models, 70(4):76-86, jul 2008.

[58] T Dokken, T Lyche, and K F Pettersen. Polynomial splines over locally refined boxpartitions. Computer Aided Geometric Design, 30(3):331-356, mar 2013.

[59] C Politis, A I Ginnis, P D Kaklis, K Belibassakis, and C Feurer. An isogeometric BEM for exterior potential-flow problems in the plane. In 2009 SIAM/ACM Joint Conference on 
Geometric and Physical Modeling, SPM '09, pages 349-354, New York, NY, USA, 2009. ACM.

[60] $\mathrm{J} \mathrm{Gu}, \mathrm{J}$ Zhang, and $\mathrm{G} \mathrm{Li.} \mathrm{Isogeometric} \mathrm{analysis} \mathrm{in} \mathrm{BIE} \mathrm{for} \mathrm{3-D} \mathrm{potential} \mathrm{problem.}$ Engineering Analysis with Boundary Elements, 36(5):858-865, 2012.

[61] K Li and X Qian. Isogeometric analysis and shape optimization via boundary integral. Computer-Aided Design, 43(11):1427-1437, 2011.

[62] L Heltai, M Arroyo, and A DeSimone. Nonsingular Isogeometric Boundary Element Method for Stokes Flows in 3D. http://dx.doi.org/10.1007/s00466-006-0084-3, 2012.

[63] M J Peake, J Trevelyan, and G Coates. Extended isogeometric boundary element method (XIBEM) for two-dimensional Helmholtz problems. Computer Methods in Applied Mechanics and Engineering, 259(0):93-102, 2013.

[64] R N Simpson, M A Scott, M Taus, D C Thomas, and H Lian. Acoustic isogeometric boundary element analysis. Computer Methods in Applied Mechanics and Engineering, page accepted, 2013.

[65] Y Wang, D J Benson, and A P Nagy. A multi-patch nonsingular isogeometric boundary element method using trimmed elements. Computational Mechanics, 56(1):173-191, 2015.

[66] G Beer, B Marussig, and J Zechner. A simple approach to the numerical simulation with trimmed CAD surfaces. Computer Methods in Applied Mechanics and Engineering, 285:776-790, mar 2015.

[67] M Feischl, G Gantner, and D Praetorius. Reliable and efficient a posteriori error estimation for adaptive $\{$ IGA $\}$ boundary element methods for weakly-singular integral equations. Computer Methods in Applied Mechanics and Engineering, 290:362-386, 2015.

[68] B Marussig, J Zechner, G Beer, and T P Fries. Fast isogeometric boundary element method based on independent field approximation. Computer Methods in Applied Mechanics and Engineering, 284:458-488, 2015.

[69] D Arnold and J Saranen. On the Asymptotic Convergence of Spline Collocation Methods for Partial Differential Equations. SIAM Journal on Numerical Analysis, 21(3):459-472, 1984. 
[70] P Juhl. A note on the convergence of the direct collocation boundary element method. Journal of Sound and Vibration, 212(4):703-719, 1998.

[71] M Taus, G J Rodin, and T J R Hughes. Isogeometric analysis of boundary integral equations. ICES report 15-12, 2015.

[72] C V Verhoosel, M A Scott, R de Borst, and T J R Hughes. An isogeometric approach to cohesive zone modeling. International Journal for Numerical Methods in Engineering, $87(1-5): 336-360,2011$.

[73] L Piegl and W Tiller. The NURBS book. springer, 1995.

[74] J C F Telles. A self-adaptive co-ordinate transformation for efficient numerical evaluation of general boundary element integrals. International Journal for Numerical Methods in Engineering, 24(5):959-973, 1987.

[75] T J Rudolphi. The use of simple solutions in the regularization of hypersingular boundary integral equations. Math. Comput. Model., 15(3-5):269-278, jan 1991.

[76] Y Liu and T J Rudolphi. Some identities for fundamental solutions and their applications to weakly-singular boundary element formulations. Engineering Analysis with Boundary Elements, 8(6):301-311, 1991.

[77] E Lutz, A R Ingraffea, and L J Gray. Use of 'simple solutions' for boundary integral methods in elasticity and fracture analysis. International Journal for Numerical Methods in Engineering, 35(9):1737-1751, 1992.

[78] Y X Mukherjee, K Shah, and S Mukherjee. Thermoelastic fracture mechanics with regularized hypersingular boundary integral equations. Engineering Analysis with Boundary Elements, 23(1):89-96, 1999.

[79] M Tanaka, V Sladek, and J Sladeck. Regularization Techniques Applied to Boundary Element Methods. Applied Mechanics Reviews, 47(10):457-499, 1994.

[80] M Guiggiani, G Krishnasamy, T J Rudolphi, and F J Rizzo. A General Algorithm for the Numerical Solution of Hypersingular Boundary Integral Equations. Journal of Applied Mechanics, 59(3):604-614, 1992. 
[81] R Simpson and J Trevelyan. A partition of unity enriched dual boundary element method for accurate computations in fracture mechanics. Computer Methods in Applied Mechanics and Engineering, 200(1-4):1-10, 2011.

[82] J M Melenk and I Babuška. The partition of unity finite element method: Basic theory and applications. Computer Methods in Applied Mechanics and Engineering, 139(1-4):289$314,1996$.

[83] N Sukumar, D L Chopp, E Béchet, and N Moës. Three-dimensional non-planar crack growth by a coupled extended finite element and fast marching method. International Journal for Numerical Methods in Engineering, 76(5):727-748, 2008.

[84] N Moës, A Gravouil, and T Belytschko. Non-planar 3D crack growth by the extended finite element and level sets-Part I: Mechanical model. International Journal for Numerical Methods in Engineering, 53(11):2549-2568, 2002.

[85] A Gravouil, N Moës, and T Belytschko. Non-planar 3D crack growth by the extended finite element and level sets-Part II: Level set update. International Journal for Numerical Methods in Engineering, 53(11):2569-2586, 2002.

[86] S Bordas and B Moran. Enriched finite elements and level sets for damage tolerance assessment of complex structures. Engineering Fracture Mechanics, 73(9):1176-1201, 2006.

[87] S Bordas, P V Nguyen, C Dunant, A Guidoum, and H Nguyen-Dang. An extended finite element library. International Journal for Numerical Methods in Engineering, 71(6):703$732,2007$.

[88] E Wyart, M Duflot, D Coulon, P Martiny, T Pardoen, J.-F. Remacle, and F Lani. Substructuring FE-XFE approaches applied to three-dimensional crack propagation. Journal of Computational and Applied Mathematics, 215(2):626-638, 2008.

[89] O A González-Estrada, J J Ródenas, E Nadal, S P A Bordas, and P Kerfriden. Equilibrated patch recovery for accurate evaluation of upper error bounds in quantities of interest. Adaptive Modeling and Simulation. Proceedings of V ADMOS, 2011.

[90] F Auricchio, L B D Veiga, T J R Hughes, A Reali, and G Sangalli. Isogeometric collocation methods. Mathematical Models and Methods in Applied Sciences, 20(11):2075-2107, 2010. 
[91] P A Martin and F J Rizzo. Hypersingular integrals: how smooth must the density be? International Journal for Numerical Methods in Engineering, 39(4):687-704, 1996.

[92] J W Eischen. An improved method for computing the J2 integral. Engineering Fracture Mechanics, 26(5):691-700, 1987.

[93] F Erdogan and G Sih. On the crackextension in plates under plane loading and transverse shear. Journal of Basic Engineering, 85:519-527, 1963.

[94] R LaGreca, M Daniel, and A Bac. Local deformation of NURBS curves. Mathematical methods for curves and surfaces, Tromso 2004, pages 243-252, 2005.

[95] A Paluszny and R W Zimmerman. Numerical fracture growth modeling using smooth surface geometric deformation. Engineering Fracture Mechanics, 108(0):19-36, 2013.

[96] H M Westergaard. Bearing pressures and cracks. Journal of Applied Mechanics, 6:A49A53, 1939.

[97] D J Smith, M R Ayatollahi, and M J Pavier. The role of T-stress in brittle fracture for linear elastic materials under mixed-mode loading. Fatigue 6 Fracture of Engineering Materials 63 Structures, 24(2):137-150, 2001.

[98] B Cotterell and J R Rice. Slightly curved or kinked cracks. International Journal of Fracture, 16(2):155-169, 1980.

[99] A R Ingraffea and M Grigoriu. Probabilistic fracture mechanics: A validation of predictive capability. Department of Structure Engineering, Cornell University, Rep. 90-8, 1990.

[100] G Ventura, J X Xu, and T Belytschko. A vector level set method and new discontinuity approximations for crack growth by EFG. International Journal for Numerical Methods in Engineering, 54(6):923-944, 2002. 